EPJ manuscript No.

(will be inserted by the editor)

\title{
Use of harmonic inversion techniques in the periodic orbit quantization of integrable systems
}

\author{
Kirsten Weibert, Jörg Main, and Günter Wunner \\ Institut für Theoretische Physik und Synergetik, Universität Stuttgart, D-70550 Stuttgart, Germany
}

November 15, 2018

\begin{abstract}
Harmonic inversion has already been proven to be a powerful tool for the analysis of quantum spectra and the periodic orbit orbit quantization of chaotic systems. The harmonic inversion technique circumvents the convergence problems of the periodic orbit sum and the uncertainty principle of the usual Fourier analysis, thus yielding results of high resolution and high precision. Based on the close analogy between periodic orbit trace formulae for regular and chaotic systems the technique is generalized in this paper for the semiclassical quantization of integrable systems. Thus, harmonic inversion is shown to be a universal tool which can be applied to a wide range of physical systems. The method is further generalized in two directions: Firstly, the periodic orbit quantization will be extended to include higher order $\hbar$ corrections to the periodic orbit sum. Secondly, the use of cross-correlated periodic orbit sums allows us to significantly reduce the required number of orbits for semiclassical quantization, i.e., to improve the efficiency of the semiclassical method. As a representative of regular systems, we choose the circle billiard, whose periodic orbits and quantum eigenvalues can easily be obtained.
\end{abstract}

PACS. 03.65.Sq Semiclassical theories and applications

\section{Introduction}

A question of fundamental interest for systems with both regular and chaotic dynamics is how quantum mechanical eigenvalues can be obtained by quantization of classical orbits. The EBK torus quantization method of Einstein, Brillouin, and Keller [1,2,3] is restricted to integrable sys' tems, i.e., the method cannot be generalized to systems with an underlying chaotic or mixed regular-chaotic dynamics [1]. Furthermore, EBK quantization requires the knowledge of all the constants of motion, which are not normally given in explicit form, and therefore practical EBK quantization based on the direct or indirect numerical construction of the constants of motion turns out to be a formidable task [4. As an alternative, EBK quantization was recast as a sum over all periodic orbits of a given topology on respective tori by Berry and Tabor [5]. In contrast to EBK-quantization, periodic orbit theory can be applied to systems with more general classical dynamics: Gutzwiller's trace formula [6, 7] for chaotic systems and the corresponding Berry-Tabor formula for regular systems [5] give the semiclassical approximation for the density of states as a sum over the periodic orbits of the underlying classical system. However, a fundamental problem of these periodic orbit sums is that they usually do not converge, or if they do, the convergence is extremely slow. During recent years, various techniques have been developed to overcome this problem. Most of them are especially designed for chaotic systems [8, 9, 10] and can- not be applied to systems with regular or mixed regularchaotic dynamics, or they depend on special properties of the system such as the existence of a symbolic dynamics. They are therefore restricted to a relatively small number of physical systems. It would be desirable to have a method at hand which is universal in the sense that it is applicable for all types of underlying classical dynamics.

Recently, a method for periodic orbit quantization, based on harmonic inversion of a semiclassical signal has been developed and successfully applied to classically chaotic systems [11, 12, 13]. The aim of the present paper is to demonstrate that this technique is equally powerful in reproducing the spectra of regular systems. The semiclassical quantization of integrable and chaotic systems on an equal footing will be the basis for applications to systems with even more general, i.e., mixed regularchaotic dynamics [14]. Furthermore, the harmonic inversion technique is generalized in two directions: Firstly, the periodic orbit quantization will be extended to include higher order $\hbar$ corrections [15], and, secondly, the use of cross-correlated periodic orbit sums [16, 17, 18] allows us to significantly reduce the required number of orbits for semiclassical quantization, i.e., to improve the efficiency of the semiclassical method. As a representative of regular systems, we choose the circle billiard whose periodic orbits and quantum eigenvalues can easily be obtained. The paper is organized as follows:

In Section 2 we give a brief overview over the periodic orbit theory for integrable systems, especially the Berry- 
Tabor formula, which is the analogue for integrable systems to Gutzwiller's trace formula for chaotic systems. We then calculate the explicit expression for the density of states of the circle billiard from the Berry-Tabor formula. The equations are generalized in two directions, firstly, to the density of states weighted with the diagonal matrix elements of one or more given operators [16], and, secondly, to include higher order $\hbar$ corrections in the periodic orbit sum 15.

The high precision analysis of quantum spectra and the method for the analytic continuation of non-convergent periodic orbit sums applied in this paper are based on the harmonic inversion of time signals. In Section 3 we briefly introduce harmonic inversion by filterdiagonalization [19,20. We also discuss an extension of the filter-diagonalization method to cross-correlation functions [19,21,22], which can be used to extract semiclassical eigenvalues and matrix elements from cross-correlated periodic orbit sums with a significantly reduced set of periodic orbits 17.

Harmonic inversion circumvents the uncertainty principle of the conventional Fourier transform and can be used for the high precision analysis of quantum spectra [13,23. In Section the method will be applied to the quantum spectra of the circle billiard. The analysis will verify the validity of the Berry-Tabor formula and its generalization to spectra weighted with diagonal matrix elements discussed in Section 2.3. Furthermore, harmonic inversion will be applied to determine the higher order $\hbar$ contributions to the periodic orbit sum. The Gutzwiller and the Berry-Tabor formula are only the leading order contributions of an expansion of the density of states in terms of $\hbar$ and therefore only yield semiclassical approximations to the eigenvalues. By analyzing the difference spectrum between exact and semiclassical eigenvalues, first order $\hbar$ corrections to the periodic orbit sum can be determined, as we will demonstrate in Section 4.2. The results are compared with the analytic expressions for the $\hbar$ expansion of the periodic orbit sum given in Section 2.4.

In Section 5, we turn to the periodic orbit quantization of integrable systems. Firstly, we show how in general the problem of extracting semiclassical eigenvalues from periodic orbit sums can be reformulated as a harmonic inversion problem: A semiclassical signal is constructed from the periodic orbit sum, the analysis of which yields the semiclassical eigenvalues of the system. The general formulae are then applied to the circle billiard and the results are compared to the exact quantum and the EBK eigenvalues. In Section 5.3 it is demonstrated how the accuracy of the semiclassical eigenvalues can be significantly improved with the help of higher order $\hbar$ corrections to the periodic orbit sum.

In Section 5.4 we address the question of how to improve the efficiency of the semiclassical quantization method, i.e., how to extract the same number of eigenvalues with a reduced set of periodic orbits, which is important especially when the orbits must be searched numerically. This is achieved by constructing cross-correlated periodic orbit sums as introduced in Section 2.3 which are then harmonically inverted with the generalized filterdiagonalization method of Section 3.2. The efficiency of the method will be discussed for various sets of operators and various sizes of the cross-correlation matrix. It is also possible to include higher order $\hbar$ corrections in the cross-correlation signal which will allow us to calculate $\hbar$ corrections even for nearly degenerate states.

Some concluding remarks are given in Section 6 .

\section{Periodic orbit theory for integrable systems}

\subsection{EBK quantization and Berry-Tabor formula}

Integrable systems are characterized by the property that their dynamics can be expressed in action-angle variables. The action variables, which are defined on certain "irreducible" paths, are constants of motion. In the $2 n$-dimensional phase space, the motion of an integrable system is restricted to $n$-dimensional tori, which are given by the values of the action variables.

A well-established method for the semiclassical quantization of integrable systems is the EBK torus quantization scheme, which was developed by Einstein, Brillouin and Keller [1, 2, 3]. In the EBK theory, the energy eigenvalues of the system are directly associated with certain classical tori. These tori are defined by the EBK conditions, which select special sets from all possible values of the action variables of the system. Each such set corresponds to a quantum mechanical eigenstate of the system. The tori selected by the EBK conditions are usually not rational, i.e., the orbits on these tori are usually not periodic.

For many physical systems the application of the EBK quantization scheme is a nontrivial task. Especially for non-separable or near-integrable systems the irreducible paths are difficult to find. Most importantly, as already discussed by Einstein [1] the torus quantization scheme cannot be extended to chaotic systems. For chaotic systems, Gutzwiller derived a semiclassical expression for the density of states in terms of the periodic orbits of the corresponding classical system: The semiclassical density of states consists of a smooth background and an oscillating part

$$
\rho(E)=\rho_{0}(E)+\rho^{\mathrm{osc}}(E)
$$

with

$\rho^{\mathrm{osc}}(E)=\frac{1}{\pi \hbar} \sum_{\mathrm{po}} \frac{T_{\mathrm{po}}}{r\left|\operatorname{det}\left(M_{\mathrm{po}}-\mathbf{1}\right)\right|^{1 / 2}} \cos \left(\frac{S_{\mathrm{po}}}{\hbar}-\mu_{\mathrm{po}} \frac{\pi}{2}\right)$.

The sum runs over all periodic orbits (po) of the system, including multiple traversals. Here, $T$ and $S$ are the period and the action of the orbit, $M$ and $\mu$ are the Monodromy matrix and the Maslov index, and the repetition number $r$ counts the traversals of the underlying primitive orbit.

For integrable systems an analogous formula for the density of states in terms of a smooth part and oscillating periodic orbit contributions was derived by Berry and Tabor [5]. While in chaotic systems the periodic orbits are isolated, the periodic orbits of integrable systems are 
all those orbits lying on rational tori - i.e., tori on which the frequencies of the motion are commensurable - and thus are non-isolated. The Berry-Tabor formula gives the density of states in terms of the rational tori:

$$
\rho(E)=\rho_{0}(E)+\rho^{\mathrm{osc}}(E)
$$

with

$$
\rho^{\mathrm{osc}}(E)=\frac{2}{\hbar^{\frac{1}{2}(n+1)}} \sum_{\mathbf{M}} \frac{\cos \left(S_{\mathbf{M}} / \hbar-\frac{1}{2} \pi \boldsymbol{\alpha} \cdot \mathbf{M}+\frac{1}{4} \pi \beta_{\mathbf{M}}\right)}{|\mathbf{M}|^{\frac{1}{2}(n-1)}\left|\boldsymbol{\omega}_{\mathbf{M}}\right|\left|K\left(\mathbf{I}_{\mathbf{M}}\right)\right|^{\frac{1}{2}}} .
$$

The sum runs over all rational tori at energy $E$, characterized by the frequency ratios given by the ray of integer numbers $\mathbf{M}$. The sum includes cases where the $M_{i}$ are not relatively prime, $\mathbf{M}=r \boldsymbol{\mu}$, which corresponds to multiple traversals of the primitive periodic orbits on the torus characterized by $\boldsymbol{\mu}$. Here, $n$ is the dimension of the system, $\mathbf{I}_{\mathbf{M}}$ and $\boldsymbol{\omega}_{\mathbf{M}}$ are the values of the action variables and the frequencies on the torus, $S_{\mathrm{M}}$ is the action of the periodic orbits on the torus, and $K$ is the scalar curvature of the energy contour. The components of $\boldsymbol{\alpha}$ are the Maslov indices of the irreducible paths on which the action variables are defined, and the phase $\beta$ is obtained from the second derivative matrix of the action variables in terms of the coordinates. In contrast to the EBK torus quantization, there is no direct relation between the eigenvalues of the system and the tori which enter the Berry-Tabor formula.

Both the EBK torus quantization and the Berry-Tabor formula are semiclassical theories delivering lowest order $\hbar$ approximations to the exact quantum eigenvalues. In general, the results of the two approaches can only be expected to be the same in lowest order of $\hbar$ but not necessarily beyond. However, it was shown in Ref. 24 that for the circle billiard, which will be discussed in the following sections, the two approaches are in fact equivalent and should yield exactly the same results.

Eq. (1) can be simplified for the special case $n=2$, i.e., for two-dimensional regular systems 25:

$\rho^{\mathrm{osc}}(E)=\frac{1}{\pi \hbar^{3 / 2}} \sum_{\mathbf{M}} \frac{T_{\mathbf{M}}}{M_{2}^{3 / 2}\left|g_{E}^{\prime \prime}\right|^{1 / 2}} \cos \left(\frac{S_{\mathbf{M}}}{\hbar}-\eta_{\mathbf{M}} \frac{\pi}{2}-\frac{\pi}{4}\right)$

The sum runs over all rational tori at energy $E$, characterized by the frequency ratio given by the integer numbers $\mathbf{M}=\left(M_{1}, M_{2}\right)$, including multiple traversals (i.e., cases where $M_{1}, M_{2}$ are not relatively prime). Here, $T_{\mathbf{M}}$ is the traversal time, and $g_{E}$ is the function describing the energy surface: $H\left(I_{1}, I_{2}=g_{E}\left(I_{1}\right)\right)=E$, where $I_{1}$ and $I_{2}$ are the action variables. The Maslov index $\eta_{\mathbf{M}}$ is obtained from the Maslov indices $\alpha_{1}, \alpha_{2}$ of the paths on which the action variables are defined:

$$
\eta_{\mathbf{M}}=\left(M_{1} \alpha_{1}+M_{2} \alpha_{2}\right)-\Theta\left(g_{E}^{\prime \prime}\right),
$$

where $\Theta$ is the Heaviside step function.

The semiclassical density of states can be expressed in terms of the semiclassical response function $g(E)$ :

$$
\rho(E)=-\frac{1}{\pi} \operatorname{Im} g(E) .
$$

For both chaotic and regular systems the response function is of the form

$$
g(E)=g_{0}(E)+\sum_{\text {po }} \mathcal{A}_{\mathrm{po}} e^{\frac{i}{\hbar} S_{\mathrm{po}}}
$$

where the amplitudes are given by the Gutzwiller or the Berry-Tabor formula, respectively.

In practical applications both the Gutzwiller formula (2) and the Berry-Tabor formula (4) suffer from the property that the periodic orbit sums usually do not converge. Depending on the system in question, this problem may be overcome, e.g., by convolution of the periodic orbit sum with a suitable averaging function [24]. But even then the convergence will usually be slow, and a large number of orbits has to be included in order to obtain the semiclassical eigenvalues. In the following sections, we will demonstrate how the convergence problem can be circumvented by the harmonic inversion method and the eigenvalues can be calculated from a relatively small number of periodic orbits.

\subsection{Application to the circle billiard}

We now apply the Berry-Tabor formula to the circle billiard as a specific separable system with two degrees of freedom.

For completeness and comparisons with the results from periodic orbit theory we first briefly review the quantum mechanical expressions and the EBK quantization condition. The exact quantum mechanical energy eigenvalues of the circle billiard with radius $R$ are given by the condition

$$
J_{m}(k R)=0, \quad m \in \mathbb{Z}, \quad E=\frac{\hbar^{2} k^{2}}{2 M},
$$

where $J_{m}$ are the Bessel functions of integer order. Here, $M$ denotes the mass of the particle, $E$ is the energy, and $k=\sqrt{2 M E} / \hbar$ is the wavenumber. The corresponding wave functions are given by

$$
\psi(r, \varphi)=J_{m}(k r) e^{i m \varphi}
$$

As $J_{-m}(x)=(-1)^{m} J_{m}(x)$, all energy eigenvalues belonging to nonzero angular momentum quantum number $(m \neq 0)$ are twofold degenerate. In the following the exact quantum mechanical results for the circle billiard are used as a benchmark for the development and application of semiclassical quantization methods for integrable systems.

The circle billiard problem in two dimensions is separable in polar coordinates. The semiclassical expressions for both EBK torus quantization and the Berry-Tabor formula for the density of states are based on the action-angle variables associated with the angular $\varphi$-motion and the radial $r$-motion 24,26]. The action variables are given by

$$
I_{\varphi}=\frac{1}{2 \pi} \oint p_{\varphi} d \varphi=L
$$




$$
\begin{aligned}
I_{r} & =\frac{1}{2 \pi} \oint p_{r} d r \\
& =\frac{1}{\pi}\left(\sqrt{2 M E R^{2}-L^{2}}-|L| \arccos \frac{|L|}{\sqrt{2 M E} R}\right)
\end{aligned}
$$

where $E$ and $L$ are the energy and the angular momentum, respectively. Quantization of the action variables

$$
\begin{aligned}
& I_{\varphi}=\left(m+\frac{\alpha_{\varphi}}{4}\right) \hbar, \quad m \in \mathbb{Z} \\
& I_{r}=\left(n+\frac{\alpha_{r}}{4}\right) \hbar, \quad n=0,1,2, \ldots
\end{aligned}
$$

with $\alpha_{\varphi}=0$ and $\alpha_{r}=3$ for the circle billiard yields the EBK quantization condition

$$
\sqrt{(k R)^{2}-m^{2}}-|m| \arccos \frac{|m|}{k R}=\left(n+\frac{3}{4}\right) \pi,
$$

where $L=m \hbar$ are the angular momentum eigenvalues.

The frequencies of the classical motion on the twodimensional tori are given by

$$
\begin{aligned}
& \omega_{\varphi}=\frac{\partial E}{\partial I_{\varphi}}=\frac{2 E}{\sqrt{2 M E R^{2}-L^{2}}} \arccos \frac{|L|}{\sqrt{2 M E} R} \\
& \omega_{r}=\frac{\partial E}{\partial I_{r}}=\frac{2 \pi E}{\sqrt{2 M E R^{2}-L^{2}}} .
\end{aligned}
$$

The Berry-Tabor formula includes all tori with a rational frequency ratio, i.e., tori on which the orbits are periodic. In the case of the circle billiard, the rational tori are given by the condition

$$
\frac{\omega_{\varphi}}{\omega_{r}}=\frac{M_{\varphi}}{M_{r}}
$$

with positive integers $M_{r}, M_{\varphi}$ and the restriction

$$
M_{r} \geq 2 M_{\varphi}
$$

The periodic orbits of the circle billiard have the form of regular polygons. The numbers $M_{r}$ and $M_{\varphi}$ can be shown to be identical with the number of sides of the corresponding polygon and its number of turns around the center of the circle, respectively [27]. Some examples are given in Figure 1. A pair of numbers $\left(M_{r}, M_{\varphi}\right)$ which are not relatively prime corresponds to multiple traversals of a primitive periodic orbit.

The classical action of the periodic orbits is given by

$$
\begin{aligned}
S_{\mathbf{M}} & =2 \pi M_{\varphi} I_{\varphi}^{(\mathrm{M})}+2 \pi M_{r} I_{r}^{(\mathbf{M})} \\
& =\sqrt{2 M E} R 2 M_{r} \sin \left(\frac{M_{\varphi}}{M_{r}} \pi\right) .
\end{aligned}
$$

As in all billiard systems, the action scales like

$$
S / \hbar=w s
$$

here with the scaling parameter

$$
w \equiv \sqrt{2 M E} R / \hbar=k R
$$

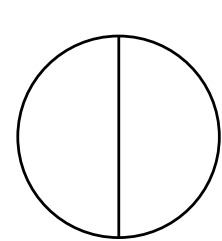

$(2,1)$

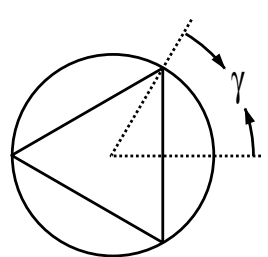

$(3,1)$

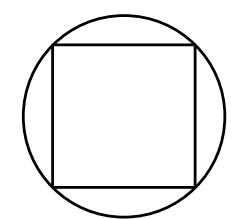

$(4,1)$

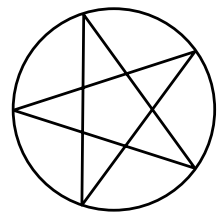

$(5,2)$

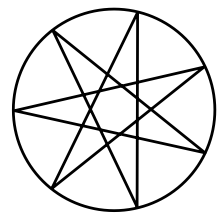

$(7,3)$
Fig. 1. Some examples of periodic orbits of the circle billiard. The orbits are labeled with the numbers $\left(M_{r}, M_{\varphi}\right)$ which correspond to the number of sides of the polygons and the number of turns around the center. The angle $\gamma$ is given by $\gamma=\pi M_{\varphi} / M_{r}$.

and the scaled action

$$
s \equiv 2 M_{r} \sin \left(\frac{M_{\varphi}}{M_{r}} \pi\right) .
$$

The form of the corresponding classical trajectory is independent of $w$. For the circle billiard with unit radius $R=1$, the scaling parameter $w$ is identical with the wavenumber $k$, and the scaled action is the length of the orbit.

For the semiclassical density of states, we start from the special version of the Berry-Tabor formula presented in Eq. (5). Using the relation

$$
\rho(w)=\frac{d E}{d w} \rho(E)
$$

valid for billiard systems, we introduce the density of states depending on the scaling parameter $w$. Evaluating the different expressions in (5) for the circle billiard then finally leads to

$$
\rho^{\mathrm{osc}}(w)=-\frac{1}{\pi} \operatorname{Im} g^{\mathrm{osc}}(w)
$$

with

$$
g^{\mathrm{osc}}(w)=\sqrt{\frac{\pi}{2}} \sqrt{w} \sum_{\mathbf{M}} m_{\mathbf{M}} \frac{s_{\mathbf{M}}^{3 / 2}}{M_{r}^{2}} e^{i\left(w s_{\mathbf{M}}-\frac{3}{2} M_{r} \pi-\frac{\pi}{4}\right)},
$$

where we have used the relations $\alpha_{\varphi}=0$ and $\alpha_{r}=3$ for the Maslov indices. The sum runs over all pairs of positive integers $\mathbf{M}=\left(M_{r}, M_{\varphi}\right)$ with $M_{r} \geq 2 M_{\varphi}$. The degeneracy factor

$$
m_{\mathbf{M}}=\left\{\begin{array}{ll}
1 ; & M_{r}=2 M_{\varphi} \\
2 ; & M_{r}>2 M_{\varphi}
\end{array},\right.
$$

accounts for the fact that all trajectories with $M_{r} \neq 2 M_{\varphi}$ can be traversed in two directions. 


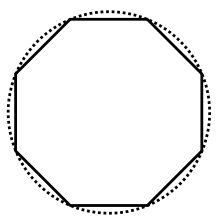

$(8,1)$

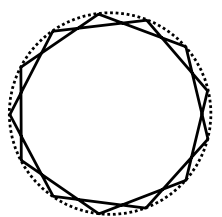

$(13,2)$

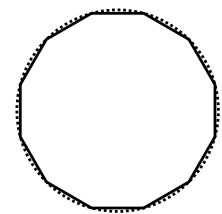

$(12,1)$

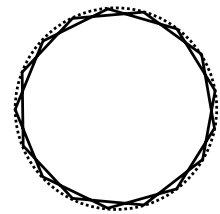

$(17,2)$

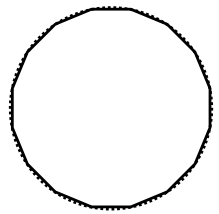

$(16,1)$

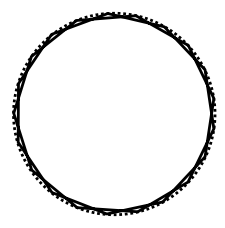

$(21,2)$
Fig. 2. Behavior of the periodic orbits of the circle billiard for large $M_{r}$. The orbits are labeled with the numbers $\left(M_{r}, M_{\varphi}\right)$. As $M_{r} \rightarrow \infty$ at constant $M_{\varphi}$, the shape of the orbit converges against $M_{\varphi}$ times the circular border of the billiard. The length of each single side of the orbits tends to zero, while the total length of the orbit $2 M_{r} \sin \left(\pi M_{\varphi} / M_{r}\right) R$ converges against $2 \pi M_{\varphi} R$ ( $R$ : radius of the billiard). The circle billiard therefore possess accumulation points of orbits at scaled actions of multiples of $2 \pi$.

Due to the rapid increase of the number of periodic orbits with growing action, the sum (26) does not converge. In our case, the problem is even more complicated by the fact that there exist accumulation points of periodic orbits at scaled actions of multiples of $2 \pi$ (see Fig. 2), which means that we cannot even include all periodic orbits up to a given finite action. In Ref. 24 the convergence problem of the sum (26) was solved by averaging it with a Gaussian function. The semiclassical eigenvalues of the circle billiard were calculated from the periodic orbit sum by including a very large number of periodic orbits. Our aim is to demonstrate that by using the harmonic inversion scheme, we can obtain eigenvalues of $w=k R$ from a relatively small number of periodic orbits. We will return to this problem in Section 5 .

\subsection{Semiclassical matrix elements}

The semiclassical trace formula for both regular and chaotic systems can be extended to include diagonal matrix elements. The calculation of individual semiclassical matrix elements is an objective in its own right. Furthermore, the extended trace formulae will allow us to construct cross-correlated periodic orbit signals and thus to significantly reduce the required number of orbits for periodic orbit quantization, as we will demonstrate in Section 5.4. Here, we will briefly recapitulate the basic ideas and equations.

Both Gutzwiller's and Berry and Tabor's formula give the semiclassical response function as a sum over contributions from periodic orbits (see Eq. (8)). The quantum mechanical response function is the trace over the Green function $G_{E}^{+}$,

$$
g_{\mathrm{qm}}(E)=\sum_{n} \frac{1}{E-E_{n}+i 0}=\operatorname{tr} G_{E}^{+} .
$$

As a generalization, one can consider the quantum mechanical response function weighted with the diagonal matrix elements of some operator $\hat{A}$, i.e.,

$$
g_{A, \mathrm{qm}}(E)=\sum_{n} \frac{\langle n|\hat{A}| n\rangle}{E-E_{n}+i 0}=\operatorname{tr}\left(G_{E}^{+} \hat{A}\right) .
$$

The semiclassical approximation to the extended response function (29) is obtained by weighting the contributions of the periodic orbits in (8) with the average $\bar{A}_{p}$ of the corresponding classical quantity $A(\mathbf{q}, \mathbf{p})$ over the periodic orbits:

$$
g_{A}(E)=g_{A, 0}(E)+\sum_{\text {po }} \mathcal{A}_{\mathrm{po}} \bar{A}_{p} e^{\frac{i}{\hbar} S_{\mathrm{po}}} .
$$

For chaotic systems, the average is taken over one period $T_{p}$ of the isolated periodic orbit 28, 29, 30:

$$
\bar{A}_{p}=\frac{1}{T_{p}} \int_{0}^{T_{p}} A(\mathbf{q}(t), \mathbf{p}(t)) d t .
$$

For an $N$-dimensional integrable system, the quantity $A$ has to be expressed in action-angle variables $(\mathbf{I}, \boldsymbol{\theta})$ and averaged over the rational torus [26]:

$$
\bar{A}_{p}=\frac{1}{(2 \pi)^{N}} \int A(\mathbf{I}, \boldsymbol{\theta}) d^{N} \theta .
$$

Eq. (29) can even be further generalized by introducing a second operator $\hat{B}$ and considering the quantity 16.

$$
g_{A B, \mathrm{qm}}(E)=\sum_{n} \frac{\langle n|\hat{A}| n\rangle\langle n|\hat{B}| n\rangle}{E-E_{n}+i 0} .
$$

If either $\hat{A}$ or $\hat{B}$ commutes with the Hamiltonian, Eq. (33) can be written as a trace formula and a calculation similar to that in 30] yields the semiclassical approximation

$$
g_{A B}(E)=g_{A B, 0}(E)+\sum_{\text {po }} \mathcal{A}_{\mathrm{po}} \bar{A}_{p} \bar{B}_{p} e^{\frac{i}{\hbar} S_{\mathrm{po}}} .
$$

Note that for general operators $\hat{A}$ and $\hat{B}$, Eq. (33) cannot be written as a trace any more. However, strong numerical evidence was provided (for both regular and chaotic systems) that Eq. (34) is correct in general, i.e., even if neither operator $\hat{A}$ nor $\hat{B}$ commutes with the Hamiltonian [16]. For chaotic systems, a mathematical proof of Eq. (34) is given in Ref. [18]. An analogous rigorous derivation for integrable systems is, to our knowledge, still lacking.

In Refs. 16, 18], the relations (33) and (34) were generalized to products of diagonal matrix elements of more than two operators. As a further extension, we can also 
introduce functions of diagonal matrix elements in the response function:

$$
g_{f(A), \mathrm{qm}}(E)=\sum_{n} \frac{f(\langle n|\hat{A}| n\rangle)}{E-E_{n}+i 0} .
$$

By a Taylor expansion of the (sufficiently smooth) function $f$ and using the results of Refs. 16, 18] for multiple products of matrix elements, we obtain the semiclassical approximation

$$
g_{f(A)}(E)=g_{f(A), 0}(E)+\sum_{\mathrm{po}} \mathcal{A}_{\mathrm{po}} f\left(\bar{A}_{p}\right) e^{\frac{i}{\hbar} S_{\mathrm{po}}} .
$$

We will use the extended trace formulae in combination with an extension of the harmonic inversion procedure to cross-correlated signals in order to significantly reduce the number of orbits which have to be included in the periodic orbit sum.

The diagonal matrix elements obtained from the extended trace formulae are semiclassical approximations to the exact quantum matrix elements. For the circle billiard, we can compare these values to those given by EBK theory. According to EBK theory, the diagonal matrix element of an operator $\hat{A}$ with respect to an eigenstate $|n\rangle$ is obtained by averaging the corresponding classical quantity $A(\mathbf{I}, \boldsymbol{\theta})$ over the quantized torus related to this eigenstate:

$$
\langle n|\hat{A}| n\rangle=\frac{1}{(2 \pi)^{N}} \int A\left(\mathbf{I}_{n}, \boldsymbol{\theta}_{n}\right) d^{N} \theta_{n}
$$

Note the difference to Eq. (32), where the average is taken over the rational tori.

\subsection{Higher order $\hbar$ corrections}

The Berry-Tabor formula for integrable systems and Gutzwiller's trace formula for chaotic systems are only the leading order terms of an expansion of the density of states in terms of $\hbar$. In billiard systems, the scaling parameter $w$ of the classical action (cf. Eq. (21)) is proportional to $\hbar^{-1}$ and thus plays the role of an inverse effective Planck constant,

$$
w=\hbar_{\mathrm{eff}}^{-1} .
$$

The $\hbar$ expansion of the response function can therefore be written as a power series in terms of $w^{-1} 15$ :

$$
g^{\mathrm{osc}}(w)=\sum_{n=0}^{\infty} g_{n}(w)=\sum_{n=0}^{\infty} \frac{1}{w^{n}} \sum_{\mathrm{po}} \mathcal{A}_{\mathrm{po}}^{(n)} e^{i s_{\mathrm{po}} w} .
$$

The zeroth order amplitudes $\mathcal{A}_{\mathrm{po}}^{(0)}$ are those of the BerryTabor or Gutzwiller formula, respectively, whereas for $n>0$, the amplitudes $\mathcal{A}_{\text {po }}^{(n)}$ give the $n^{\text {th }}$ order corrections $g_{n}(w)$ to the response function. Explicit expressions for the first order correction terms for chaotic systems were developed by Gaspard and Alonso [31, 32] and by Vattay and Rosenqvist [33, 34, 35, following two different approaches. Vattay and Rosenqvist compute the corrections by solving the local Schrödinger equation in the neighborhood of periodic orbits. They introduce a quantum generalization of the Gutzwiller formula which contains these local eigenvalues. The results of Refs. 33, 34, 35 cannot directly be applied to integrable systems, as the derivations are valid only for isolated periodic orbits. To our knowledge, a general theory for $\hbar$ corrections to the Berry-Tabor formula does not yet exist.

Nevertheless, for the circle billiard we have succeeded in obtaining an explicit expression for the first order $\hbar$ corrections to the Berry-Tabor formula. The calculations are quite lengthy and are therefore deferred to Appendix A. Our final result for the first order $\hbar$ amplitude of the circle billiard in (39) reads:

$$
\mathcal{A}_{\text {po }}^{(1)}=\mathcal{A}_{\text {po }}^{(0)} \frac{i}{2} M_{r}\left(\frac{1}{3 \sin \gamma}-\frac{5}{6 \sin ^{3} \gamma}\right),
$$

with $\gamma \equiv \pi M_{\varphi} / M_{r}$ and $\mathcal{A}_{\mathrm{po}}^{(0)}$ the zeroth order amplitudes given by the Berry-Tabor formula. Using the zeroth order amplitudes from Eq. (26), we finally obtain

$$
\mathcal{A}_{\mathrm{po}}^{(1)}=\sqrt{w} \sqrt{\pi M_{r}} \frac{2 \sin ^{2} \gamma-5}{6 \sin ^{3 / 2} \gamma} e^{-i\left(\frac{3}{2} M_{r} \pi-\frac{\pi}{4}\right)} .
$$

As explained above our derivation of Eq. (41) cannot be applied to general integrable systems. It will be an interesting task for the future to develop a general theory for the higher order $\hbar$ corrections to the Berry-Tabor formula.

\section{Harmonic inversion by filter-diagonalization}

The quantization of the periodic orbit sum as well as the analysis of quantum spectra in terms of the periodic orbits can be reformulated as a harmonic inversion problem of formulae which have been introduced in the previous Section 2. Before discussing these applications in Sections 4 and 5 we will now briefly recapitulate the basic ideas and the technical tools of harmonic inversion by filterdiagonalization. In Section 3.1 we will start with the harmonic inversion of a single function. The equations will be generalized in Section 3.2 to the harmonic inversion of cross-correlated signals.

\subsection{Harmonic inversion of a single function}

The harmonic inversion problem can be formulated as a nonlinear fit of a signal $C(t)$ to the form

$$
C(t)=\sum_{k} d_{k} e^{-i \omega_{k} t}
$$


where $d_{k}$ and $\omega_{k}$ are generally complex variational parameters. Other than, e.g., in a simple Fourier transformation of the signal, there is no restriction to the closeness of the frequencies $\omega_{k}$. Solving (42) will therefore yield a high resolution analysis of the signal $C(t)$. The signal length required for resolving the frequencies $\omega_{k}$ by harmonic inversion can be estimated to be

$$
t_{\max } \approx 4 \pi \bar{\rho}(\omega)
$$

where $\bar{\rho}(\omega)$ is the mean density of frequencies in the range of interest.

A method which has proven very useful for solving the harmonic inversion problem is the filter-diagonalization procedure [19,20]. This procedure allows us to compute the frequencies $\omega_{k}$ in any small interval $\left[\omega_{\min }, \omega_{\max }\right]$ given. The idea is to consider the signal $C(t)$ on an equidistant grid

$$
c_{n}=C(n \tau) ; \quad n=0,1,2, \ldots
$$

and to associate $c_{n}$ with an autocorrelation function of a suitable fictitious dynamical system, described by a complex symmetric effective Hamiltonian $H_{\text {eff }}$ :

$$
c_{n}=\left(\Phi_{0} \mid e^{-i n \tau H_{\mathrm{eff}}} \Phi_{0}\right) .
$$

Here, the brackets denote a complex symmetric inner product $(a \mid b)=(b \mid a)$, i.e., no complex conjugation of either $a$ or $b$. The harmonic inversion problem can then be reformulated as solving the eigenvalue problem for the effective Hamiltonian $H_{\text {eff }}$. The frequencies $\omega_{k}$ are the eigenvalues of the Hamiltonian

$$
\left.\left.H_{\mathrm{eff}} \mid \Upsilon_{k}\right)=\omega_{k} \mid \Upsilon_{k}\right)
$$

and the amplitudes are obtained from the eigenvectors $\Upsilon_{k}$ :

$$
d_{k}=\left(\Phi_{0} \mid \Upsilon_{k}\right)^{2}
$$

The filter-diagonalization method solves this eigenvalue problem in a small set of basis vectors $\Psi_{j}$. The Hamiltonian and the initial state $\Phi_{0}$ do not have to be known explicitly but are given implicitly by the quantities $c_{n}$. In detail, the procedure works as follows:

A small set of values $\varphi_{j}$ in the frequency interval of interest is chosen. The set must be larger than the number of frequencies in this interval. The values $\varphi_{j}$ are used to construct the small Fourier-type basis

$$
\Psi_{j}=\sum_{n=0}^{M} e^{i n\left(\varphi_{j}-\tau H_{\mathrm{eff}}\right)} \Phi_{0}
$$

The matrix elements of the evolution operator at a given time $p \tau$ in this basis can be expressed in terms of the quantities $c_{n}$ :

$U_{j j^{\prime}}^{(p)} \equiv\left(\Psi_{j} \mid e^{-i p \tau H_{\mathrm{eff}}} \Psi_{j^{\prime}}\right)=\sum_{n=0}^{M} \sum_{n^{\prime}=0}^{M} e^{i\left(n \varphi_{j}+n^{\prime} \varphi_{j^{\prime}}\right)} c_{n+n^{\prime}+p}$
The frequencies $\omega_{k}$ are then obtained by solving the generalized eigenvalue problem

$$
\mathbf{U}^{(p)} \mathbf{B}_{k}=e^{-i p \tau \omega_{k}} \mathbf{B}_{k}
$$

The amplitudes $d_{k}$ can be calculated from the eigenvectors and are given by

$$
d_{k}=\left(\sum_{j} B_{j k} \sum_{n=0}^{M} c_{n} e^{i n \varphi_{j}}\right)^{2}
$$

The values of $\omega_{k}$ and $d_{k}$ obtained by the above procedure should be independent of $p$. This condition can be used to identify non-converged frequencies by comparing the results for different values of $p$. The difference between the frequency values obtained for different $p$ can be used as a simple error estimate.

\subsection{Harmonic inversion of cross-correlated signals}

An important extension of the filter-diagonalization method for harmonic inversion is the generalization to cross-correlation functions 19, 21, 22, 17. This extended method allows us to significantly reduce the signal length required to resolve the frequencies contained in the signal. The idea is not to consider a single signal $C(t)$ as given in Eq. (42) but a set of cross-correlated signals

$$
C_{\alpha \alpha^{\prime}}(t)=\sum_{k} d_{\alpha \alpha^{\prime}, k} e^{-i \omega_{k} t} ; \quad \alpha, \alpha^{\prime}=1, \ldots, N
$$

with the restriction

$$
d_{\alpha \alpha^{\prime}, k}=b_{\alpha, k} b_{\alpha^{\prime}, k}
$$

This set of signals considered on an equidistant grid

$$
c_{n \alpha \alpha^{\prime}}=C_{\alpha \alpha^{\prime}}(n \tau) ; \quad n=0,1,2, \ldots
$$

is now associated with a time cross-correlation function between an initial state $\Phi_{\alpha}$ and a final state $\Phi_{\alpha^{\prime}}$ :

$$
c_{n \alpha \alpha^{\prime}}=\left(\Phi_{\alpha^{\prime}} \mid e^{-i n \tau H_{\mathrm{eff}}} \Phi_{\alpha}\right) .
$$

Again, the frequencies $\omega_{k}$ are obtained as the eigenvalues of the effective Hamiltonian $H_{\text {eff }}$. The amplitudes are now given by the relation

$$
b_{\alpha, k}=\left(\Phi_{\alpha} \mid \Upsilon_{k}\right) .
$$

In analogy to the procedure described in Section 3.1, this eigenvalue problem is solved in a small set of basis vectors $\Psi_{j \alpha}$ in order to obtain the frequencies in a given interval $\left[\omega_{\min }, \omega_{\max }\right]$.

The advantage of the above procedure becomes evident if one considers the information content of the set of signals. Due to the restriction (53), the $N \times N$ set of signals $C_{\alpha \alpha^{\prime}}(t)$ may contain $N$ independent signals, which are known to possess the same frequencies $\omega_{k}$. This means 
that, at constant signal length, the matrix may contain $N$ times as much information about the frequencies as a single signal, provided that the whole set is inverted simultaneously. On the other hand, the information content is proportional to the signal length. This means that the signal length required to resolve the frequencies in a given interval is reduced by a factor of $N$. This statement clearly holds only approximately and for small matrix dimensions $N$. However, a significant reduction of the required signal length can be achieved.

\section{High resolution analysis of quantum spectra}

Harmonic inversion is a powerful tool to calculate the classical periodic orbit contributions to the density of states from the quantum mechanical eigenvalues or from experimental spectra, thus delivering a high resolution analysis of the spectra in terms of the classical orbits. The method allows us, e.g., to resolve clusters of orbits or to discover hidden ghost orbit contributions in the spectra, which would not be resolved by conventional Fourier analysis of the spectra [13,23. Here, we will analyze the quantum spectra of the circle billiard as a representative of integrable systems. The analysis will verify the validity of the Berry-Tabor formula and its extensions to semiclassical matrix elements and higher order $\hbar$ corrections discussed in Section 2 .

\subsection{Leading order periodic orbit contributions to the}

\section{trace formula}

\subsubsection{General procedure}

In this section we develop the general procedure for the analysis of quantum spectra in terms of periodic orbits by harmonic inversion. This procedure is universal in the sense that it can be applied to both regular and chaotic systems. We will apply it to the circle billiard as a representative of regular systems in the next section.

We start from the semiclassical density of states given by the Berry-Tabor or the Gutzwiller formula. As in Section 2, we consider scaling systems where the density of states depends on the scaling parameter $w[w=k R$ for the circle billiard], i.e., $\rho(w)=-(1 / \pi) \operatorname{Im} g(w)$ with $g(w)$ the semiclassical response function. Both the Berry-Tabor and the Gutzwiller formula give the oscillating part of the response function in the form

$$
g^{\mathrm{osc}}(w)=\sum_{\mathrm{po}} \mathcal{A}_{\mathrm{po}} e^{i w s_{\mathrm{po}}}
$$

where the sum runs over all rational tori (regular systems) or all periodic orbits (chaotic systems) of the underlying classical system, respectively. Here, $S_{\mathrm{po}}$ is the action of the periodic orbit. The form of the amplitude $\mathcal{A}_{\text {po }}$ depends on whether the system is chaotic or regular and also contains phase information.

The exact quantum mechanical density of states is given by

$$
\rho_{\mathrm{qm}}(w)=\sum_{k} m_{k} \delta\left(w-w_{k}\right)
$$

where the $w_{k}$ are the exact quantum eigenvalues of the scaling parameter and the $m_{k}$ are their multiplicities. The analysis of the quantum spectrum in terms of periodic orbit contributions can now be reformulated as adjusting the exact quantum mechanical density of states (58) to the semiclassical form

$$
\begin{aligned}
\rho^{\mathrm{osc}}(w) & =-\frac{1}{\pi} \operatorname{Im} g^{\mathrm{osc}}(w) \\
& =-\frac{1}{2 \pi i} \sum_{\mathrm{po}}\left(\mathcal{A}_{\mathrm{po}} e^{i w s_{\mathrm{po}}}-\mathcal{A}_{\mathrm{po}}^{*} e^{-i w s_{\mathrm{po}}}\right) .
\end{aligned}
$$

If the amplitudes $\mathcal{A}_{\text {po }}$ do not depend on $w$, the semiclassical density of states is of the form (42) [here, with $w$ playing the role of $t$ and $s_{\text {po }}$ playing the role of $\left.\omega_{k}\right]$. That means, we have reformulated the problem of extracting the periodic contributions from the quantum spectrum as a harmonic inversion problem. In the fitting procedure, we ignore the non-oscillating, smooth part of the density of states. This part does not fulfill the ansatz (42) of the harmonic inversion method and therefore acts as a kind of noise, which will be separated from the oscillating part of the "signal" by the harmonic inversion procedure.

In practice, in order to regularize the $\delta$ functions in (58), we convolute both expressions (58) and (59) with a Gaussian function,

$$
C_{\sigma}(w)=\frac{1}{\sqrt{2 \pi} \sigma} \int_{-\infty}^{\infty} \rho\left(w^{\prime}\right) e^{-\left(w-w^{\prime}\right)^{2} / 2 \sigma^{2}} d w^{\prime}
$$

In our calculations, we usually took the convolution width to be about three times the step width $\tau$ in the signal (44). Typical values are $\tau=\Delta w=0.002$ and $\sigma=0.006$. The resulting quantum mechanical signal is

$$
C_{\mathrm{qm}, \sigma}(w)=\frac{1}{\sqrt{2 \pi} \sigma} \sum_{k} m_{k} e^{-\left(w-w_{k}\right)^{2} / 2 \sigma^{2}}
$$

and the corresponding semiclassical quantity reads

$$
C_{\sigma}(w)=-\frac{1}{2 \pi i} \sum_{\mathrm{po}}\left(\mathcal{A}_{\mathrm{po}} e^{i w s_{\mathrm{po}}}-\mathcal{A}_{\mathrm{po}}^{*} e^{-i w s_{\mathrm{po}}}\right) e^{-\frac{1}{2} \sigma^{2} s_{\mathrm{po}}^{2}} .
$$

The above procedure still works if the amplitudes in (57) are not independent of $w$ but possess a dependency of the form

$$
A_{\mathrm{po}}=w^{\alpha} a_{\mathrm{po}},
$$

which is, for example, the case for regular billiards. This dependency can be eliminated [15] by replacing the semiclassical response function $g(w)$ with the quantity

$$
g^{\prime}(w)=w^{-\alpha} g(w)=w^{-\alpha} g_{0}(w)+\sum_{\text {po }} a_{\mathrm{po}} e^{i w s_{\mathrm{po}}} .
$$


When introducing the corresponding quantum mechanical response function

$$
g_{\mathrm{qm}}^{\prime}(w)=\sum_{k} \frac{m_{k} w_{k}^{-\alpha}}{w-w_{k}+i 0}
$$

the procedure can be carried out for $\rho^{\prime}(w)=$ $(-1 / \pi) \operatorname{Im} g^{\prime}(w)$ as described above.

In addition to considering the pure density of states, the relations of Section 2.3 can be used to calculate the averages of classical quantities over the periodic orbits from the quantum diagonal matrix elements of the corresponding operators. If we start from the extended quantum response function (29), including diagonal matrix elements of some operator $\hat{A}$, the analysis of the signal should again yield the actions $s_{\text {po }}$ as frequencies but with the amplitudes weighted with the classical averages $\bar{A}_{p}$ of the corresponding classical quantities. In the same way, we can also use the extended response function (33), which includes diagonal matrix elements of two different operators.

\subsubsection{Application to the circle billiard}

For the circle billiard, the oscillating part $g^{\mathrm{osc}}(w)$ of the semiclassical response function is given by Eq. (26). If one eliminates the dependency of the amplitudes on $w$ by defining

$$
\rho^{\prime}(w)=\frac{1}{\sqrt{w}} \rho(w),
$$

the resulting expression for the density of states

$$
\begin{array}{r}
\rho^{\prime}(w)=\frac{1}{\sqrt{8 \pi}} \sum_{\mathbf{M}} m_{\mathbf{M}} \frac{s_{\mathbf{M}}^{3 / 2}}{M_{r}^{2}}\left(e^{i\left(\frac{3}{2} M_{r} \pi-\frac{\pi}{4}\right)} e^{-i w s_{\mathbf{M}}}\right. \\
\left.+e^{-i\left(\frac{3}{2} M_{r} \pi-\frac{\pi}{4}\right)} e^{i w s_{\mathbf{M}}}\right)
\end{array}
$$

is of the form (42), here with $S_{\mathbf{M}}$ playing the role of $w_{k}$. The quantum mechanical quantity corresponding to (66) is

$$
\rho_{\mathrm{qm}}^{\prime}(w)=\sum_{k} \frac{m_{k}}{\sqrt{w}_{k}} \delta\left(w-w_{k}\right) .
$$

In addition to analyzing the pure quantum spectrum of the circle billiard, we also considered spectra weighted with diagonal matrix elements of different operators (cf. Section 2.3). We used three different operators, viz.

- the absolute value of the angular momentum $L$ as an example of a constant of motion,

- the distance $r$ from the center as an example of a quantity which is no constant of motion,

- the variance of the radius $\left\langle r^{2}\right\rangle-\langle r\rangle^{2}$ as an example using the relation (34) for products of operators.

The classical angular momentum $L$ is proportional to $w$, which means that when constructing the signal for $L, g(w)$ now has to be multiplied by $w^{-3 / 2}$ instead of $w^{-1 / 2}$ (cf. Eq. (64)).
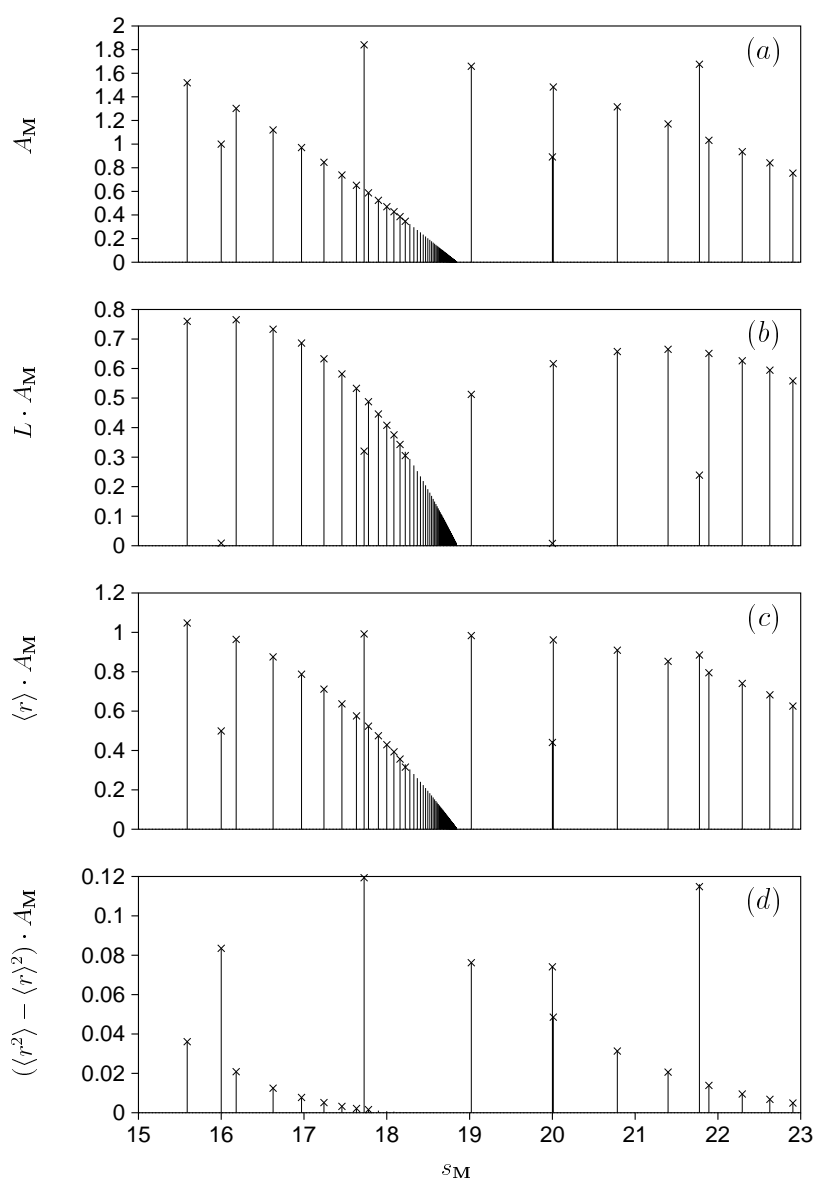

Fig. 3. Periodic orbit contributions to the trace formula calculated from the quantum spectrum of the circle billiard, including different operators. The quantities plotted are the classical amplitudes $A_{\mathrm{M}}=m_{\mathrm{M}} s_{\mathrm{M}}^{3 / 2} / M_{r}^{2}$ times the classical averages of the operators indicated, versus the scaled actions of the orbits. Crosses: values obtained from the quantum spectrum by harmonic inversion. Solid lines: values obtained from classical calculations. $L$ : angular momentum in units of $\hbar w$ ( $w$ : scaling parameter), $r$ : distance from the center in units of the radius $R$ of the billiard.

We calculated the scaled actions and classical amplitudes of the periodic orbits in the interval $s_{\mathbf{M}} \in[15,23]$. The signal was constructed from the exact zeros of the Bessel functions, up to a value of $w_{\max }=500$. The accuracy of results is improved if we cut off the lower part of the signal, using only zeros larger than $w_{\text {min }}=300$. A possible explanation for this is that the low zeros are in a sense "too much quantum" for the semiclassical periodic orbit sum.

Figure 3 shows the results of our calculation. The positions of the solid lines are the scaled actions of the classical periodic orbits, their heights are the classical amplitudes $m_{\mathrm{M}} s_{\mathrm{M}}^{3 / 2} / M_{r}^{2}$ times the respective averaged classical quantity. The crosses are the results obtained by harmonic inversion of the signal constructed from the zeros of the Bessel functions. There is an excellent agreement between the spectra, illustrating the validity of the Berry-Tabor 
formula and its extension to semiclassical matrix elements discussed in Section 2.3. The examined interval contains an accumulation point of orbits $(s=6 \pi)$. Here, only those orbits were resolved which were still sufficiently isolated.

It might be surprising that, although the Berry-Tabor formula only gives a semiclassical approximation to the density of states and we started from the exact quantum mechanical density, our results for the periodic orbit contributions are exact and do not show any deviations due to the error of the semiclassical approximation. The reason for this will become obvious in the following Section.

\subsection{Higher order $\hbar$ corrections to the trace formula}

An interesting application of the method described in the previous Section 4.1 is the determination of higher order $\hbar$ contributions to the periodic orbit sum. The higher orders can be obtained by analysis of the difference spectrum between the exact quantum and semiclassical eigenvalues, as we will show below.

As explained in Section 2.4, the Berry-Tabor formula for integrable systems as well as the Gutzwiller formula for chaotic systems are the leading order terms of an expansion of the density of states in terms of $\hbar$. For scaling systems, this expansion can be put in the form (cf. (39))

$$
g^{\mathrm{osc}}(w)=\sum_{n=0}^{\infty} g_{n}(w)=\sum_{n=0}^{\infty} \frac{1}{w^{n}} \sum_{\mathrm{po}} \mathcal{A}_{\mathrm{po}}^{(n)} e^{i s_{\mathrm{po}} w} .
$$

Provided that the amplitudes $\mathcal{A}_{\text {po }}^{(n)}$ in (69) do not depend on $w$, only the zeroth order term fulfills the ansatz (42) for the harmonic inversion procedure with constant amplitudes and frequencies. In systems like regular billiards, where the amplitudes possess a $w$ dependence of the form $\mathcal{A}_{\mathrm{po}}^{(n)}=w^{\alpha} a_{\mathrm{po}}^{(n)}$, the same argumentation holds if we consider $g^{\prime}(w)=w^{-\alpha} g(w)$ instead of $g(w)$ (cf. Section 4.1.1). This is the reason why the analysis of the quantum spectrum yields exactly the lowest order amplitudes $\mathcal{A}_{\text {po }}^{(0)}$, without any deviations due to the semiclassical error: As the higher order terms do not fulfill the ansatz, the $\mathcal{A}_{\text {po }}^{(0)}$ are the best fit for the amplitudes. The higher oder terms have similar properties as a weak noise and are separated from the "true" signal by the harmonic inversion procedure.

Although the direct analysis of the quantum spectrum only yields the lowest order amplitudes, higher order corrections can still be extracted from the spectrum by harmonic inversion. Assume that the exact eigenvalues $w_{k}$ and their $(n-1)^{\text {st }}$ order approximations $w_{k, n-1}$ are given. We can then calculate the difference between the exact quantum mechanical and the $(n-1)^{\text {st }}$ order response function

$g^{\mathrm{qm}}(w)-\sum_{j=0}^{n-1} g_{j}(w)=\sum_{j=n}^{\infty} g_{j}(w)=\sum_{j=n}^{\infty} \frac{1}{w^{j}} \sum_{\mathrm{po}} \mathcal{A}_{\mathrm{po}}^{(j)} e^{i s_{\mathrm{po}} w}$.

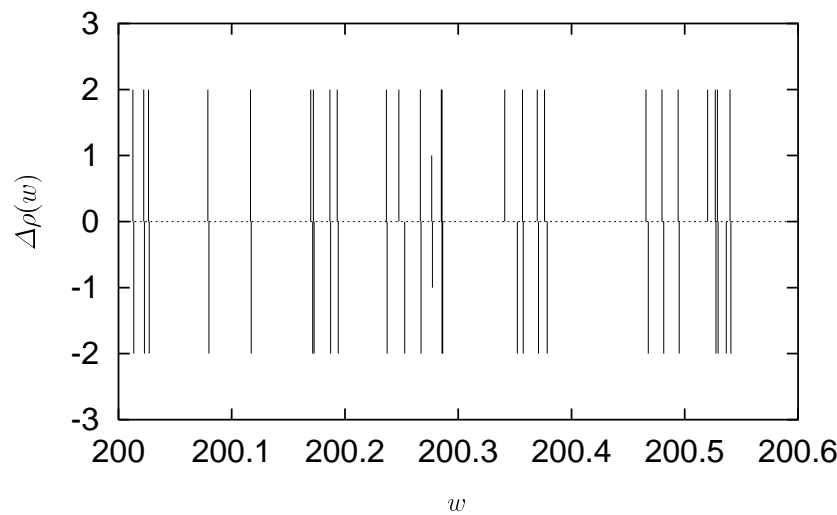

Fig. 4. Part of the difference spectrum $\Delta \rho_{\mathrm{qm}}(w)-\Delta \rho_{\mathrm{EBK}}(w)$ between the exact quantum mechanical and the semiclassical density of states. The absolute values of the peak heights mark the multiplicities of the states.

The leading order terms in (70) are $\sim w^{-n}$, i.e., multiplication with $w^{n}$ yields

$$
w^{n}\left[g^{\mathrm{qm}}(w)-\sum_{j=0}^{n-1} g_{j}(w)\right]=\sum_{\mathrm{po}} \mathcal{A}_{\mathrm{po}}^{(n)} e^{i s_{\mathrm{po}} w}+\mathcal{O}\left(\frac{1}{w}\right) .
$$

In (71) we have restored the functional form (42). The harmonic inversion of the function (71) will now provide the periods $s_{\mathrm{po}}$ and the $n^{\text {th }}$ order amplitudes $\mathcal{A}_{\mathrm{po}}^{(n)}$ of the $\hbar$ expansion (69).

In practice, we will follow the procedure outlined in Section 4.1.1 to construct a smooth signal, i.e., we consider the densities of states $\rho(w)=-(1 / \pi) \operatorname{Im} g(w)$ rather than the response functions $g(w)$, and smoothen the signal by convoluting it with a Gaussian function.

For the circle billiard, the exact quantum eigenvalues are given by the condition (9), while the zeroth order eigenvalues are equal to the EBK eigenvalues given by (15) (cf. Section 2.1). From the difference between the exact and the semiclassical density of states, we can calculate the amplitudes $\mathcal{A}_{\text {po }}^{(1)}$ of the first order correction to the trace formula.

We analyzed the difference spectrum between exact and EBK eigenvalues of the circle billiard in the range $100<w<500$. Figure 1 shows a small part of this difference spectrum. The results of the harmonic inversion of the spectrum are presented in Figure E. The crosses mark the values

$$
f(\gamma) \equiv \frac{2}{\sqrt{\pi M_{r}}} \frac{1}{\sqrt{w}}\left|\mathcal{A}_{\mathrm{po}}^{(1)}\right|
$$

with $\gamma=\pi M_{\varphi} / M_{r}$ which we obtained for the periodic orbits by harmonic inversion of the difference spectrum. The crosses are labeled with the numbers $\left(M_{r}, M_{\varphi}\right)$ of the orbits. The solid line in Fig. 5 is the theoretical curve

$$
f(\gamma)=\frac{5-2 \sin ^{2} \gamma}{3 \sin ^{3 / 2} \gamma}
$$




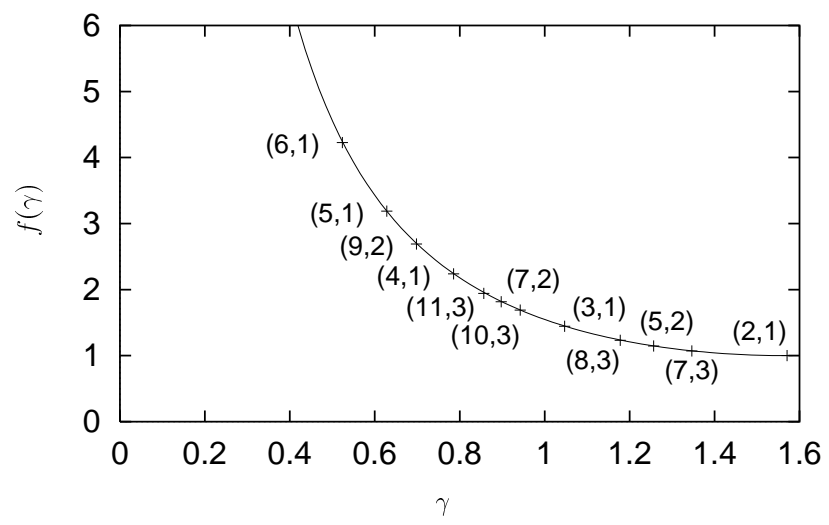

Fig. 5. First order $\hbar$ corrections to the Berry-Tabor formula for the circle billiard. Crosses: amplitudes $\left(2 / \sqrt{\pi M_{r}}\right)\left|\mathcal{A}_{\mathrm{po}}^{(1)}\right|$ obtained by harmonic inversion. The orbits are labeled by the numbers $\left(M_{r}, M_{\varphi}\right)$. Solid line: theoretical curve $f(\gamma)=$ $\left(5-2 \sin ^{2} \gamma\right) /\left(3 \sin ^{3 / 2} \gamma\right)$.

which results from our analytical expression (41) for the first order amplitudes discussed in Section 2.4. The results obtained by harmonic inversion are in excellent agreement with the theoretical curve, which clearly illustrates the validity of Eq. (41).

\section{Periodic orbit quantization}

\subsection{General procedure}

We now turn to the problem of extracting eigenvalues from the periodic orbit sum. We will demonstrate that the harmonic inversion procedure, which has already been successfully applied to extract the eigenvalues of chaotic systems [11, 12], can be used for integrable systems as well when starting from the Berry-Tabor formula.

As previously (see Section 2), we consider scaling systems and start from the response function

$$
g(w)=g_{0}(w)+\sum_{\mathrm{po}} \mathcal{A}_{\mathrm{po}} e^{i w s_{\mathrm{po}}}
$$

depending on the scaling parameter $w$. The amplitudes $\mathcal{A}_{\text {po }}$ are those of the Berry-Tabor or the Gutzwiller formula for regular and chaotic systems, respectively. The periodic orbit sum in (74) usually does not converge, or, at least, the convergence will be very slow. In practice, especially for chaotic systems, only the orbits with small scaled actions will be known. Nevertheless, the eigenvalues of the scaling parameter can still be extracted from the periodic orbit sum. The central idea is to adjust Eq. (74), with the sum including periodic orbits up to a finite action $s_{\text {max }}$, to the functional form of the corresponding quantum mechanical response function

$$
g_{\mathrm{qm}}(w)=\sum_{k} \frac{m_{k}}{w-w_{k}+i 0} .
$$

This fitting problem cannot be solved directly, but can be reformulated as a harmonic inversion problem [11,12]. The first step of the reformulation is a Fourier transformation of the response functions with respect to $w$ :

$$
C(s)=\frac{1}{2 \pi} \int_{-\infty}^{\infty} g(w) e^{-i s w} d w
$$

In the semiclassical response function, we only consider the oscillating part of $g(w)$. The smooth part, which does not possess a suitable form for the harmonic inversion method, would only give a contribution to the signal for very small $s$. Assuming that the amplitudes in (74) do not depend on $w$, the result of the Fourier transformation is

$$
\begin{aligned}
C(s) & =\sum_{\mathrm{po}} \mathcal{A}_{\mathrm{po}} \delta\left(s-s_{\mathrm{po}}\right) \\
C_{\mathrm{qm}}(s) & =-i \sum_{k} m_{k} e^{-i s w_{k}} .
\end{aligned}
$$

Like in Section 4.1.1 we convolute the signals (77) and (78) with a Gaussian function with width $\sigma$, resulting in

$$
\begin{aligned}
C_{\sigma}(s) & =\frac{1}{\sqrt{2 \pi} \sigma} \sum_{\mathrm{po}} \mathcal{A}_{\mathrm{po}} e^{-\left(s-s_{\mathrm{po}}\right)^{2} / 2 \sigma^{2}}, \\
C_{\mathrm{qm}, \sigma}(s) & =-i \sum_{k} m_{k} e^{-\frac{1}{2} \sigma^{2} w_{k}^{2}} e^{-i s w_{k}} .
\end{aligned}
$$

Typical values of the convolution width are $\sigma=0.006$ for signals with step width $\Delta s=0.002$. The eigenvalues of the scaling parameter are now obtained by adjusting the signal $C_{\sigma}(s)$ to $(80)$, which is of the functional form (42). The frequencies $w_{k}$ obtained by harmonic inversion of the signal (79) are the eigenvalues of the scaling parameter $w$; from the amplitudes $d_{k}$, the multiplicities $m_{k}$ can be calculated.

Like the general procedure for analyzing quantum spectra (see Section 4.1.1), the above procedure still works if the amplitudes in (74) are not independent of $w$ but possess a dependency of the form

$$
\mathcal{A}_{\mathrm{po}}=w^{\alpha} a_{\mathrm{po}}
$$

We can again eliminate this dependency by replacing $g(w)$ with the quantity

$$
g^{\prime}(w)=w^{-\alpha} g(w) .
$$

The semiclassical signal is then given by

$$
C_{\sigma}(s)=\frac{1}{\sqrt{2 \pi} \sigma} \sum_{\mathrm{po}} a_{\mathrm{po}} e^{-\left(s-s_{\mathrm{po}}\right)^{2} / 2 \sigma^{2}}
$$

and the corresponding quantum mechanical signal reads

$$
C_{\mathrm{qm}, \sigma}(s)=-i \sum_{k} m_{k} w_{k}^{-\alpha} e^{-\frac{1}{2} \sigma^{2} w_{k}^{2}} e^{-i s w_{k}} .
$$




\subsection{Semiclassical eigenvalues of the circle billiard}

\subsubsection{Construction of the periodic orbit signal}

The semiclassical response function of the circle billiard is given by Eq. (26). The amplitudes in (26) are proportional to $w^{1 / 2}$. As described in Section 5.1, we eliminate this dependency on $w$ by introducing the quantity

$$
g^{\prime}(w)=w^{-1 / 2} g(w)
$$

Applying Eqs. (83) and (84), we now obtain the semiclassical and the corresponding exact quantum signal for the circle billiard:

$$
\begin{aligned}
C_{\sigma}(s) & =\frac{e^{-i \frac{\pi}{4}}}{2 \sigma} \sum_{\mathbf{M}} m_{\mathbf{M}} \frac{s_{\mathbf{M}}^{3 / 2}}{M_{r}^{2}} e^{-i \frac{3}{2} M_{r} \pi} e^{-\left(s-s_{\mathbf{M}}\right)^{2} / 2 \sigma^{2}}, \\
C_{\mathrm{qm}, \sigma}(s) & =-i \sum_{k} \frac{m_{k}}{\sqrt{w}_{k}} e^{-\frac{1}{2} \sigma^{2} w_{k}^{2}} e^{-i s w_{k}} .
\end{aligned}
$$

Eq. (87) possesses the functional form (42) with

$$
d_{k}=-i \frac{m_{k}}{\sqrt{w}_{k}} e^{-\frac{1}{2} \sigma^{2} w_{k}^{2}} .
$$

Applying the harmonic inversion method to the signal (86) should yield the eigenvalues of $w$ as frequencies, with the amplitudes given by Eq. (88).

\subsubsection{Results for the lowest eigenvalues}

We calculated the eigenvalues of the scaling parameter $w=k R$ for the lowest states of the circle billiard from a signal of length $s_{\max }=150$. For the construction of the signal, we chose a minimum length for the sides of the periodic orbits as cut-off criterion at the accumulation points (cf. Fig. 2). We observed that the results were nearly independent of the choice of this parameter, as long as the minimum length was not chosen too large.

Table 1 presents the semiclassical eigenvalues $w_{\text {hi }}$ and multiplicities $m_{\mathrm{hi}}$ obtained by harmonic inversion of the periodic orbit signal (86). For comparison, the exact quantum mechanical and the EBK results are also given in Table 1. The eigenvalues obtained by harmonic inversion clearly reproduce the EBK eigenvalues within an accuracy of $10^{-4}$ or better. The deviation of the $w_{\text {hi }}$ from the EBK eigenvalues is significantly smaller than the error of the semiclassical approximation. The improvement of the semiclassical quantization by including higher order $\hbar$ corrections to the periodic orbit sum will be discussed in Section 5.3.

Note that for calculating the eigenvalues of the circle billiard by a direct evaluation of the periodic orbit sum, a huge number of periodic orbit terms is required, e.g., orbits with maximum length $s_{\max }=30000$ were included
Table 1. Lowest eigenvalues $w_{k}$ and multiplicities $m_{k}$ of the scaling parameter $w=k R$ of the circle billiard. $w_{\mathrm{ex}}$ and $m_{\mathrm{ex}}$ : exact quantum values. $w_{\mathrm{EBK}}$ : EBK eigenvalues. $w_{\mathrm{hi}}$ and $m_{\mathrm{hi}}$ : values obtained by harmonic inversion of a signal of length $s_{\max }=150$. The numbers $n$ and $m$ are the radial and angular momentum quantum numbers. The nearly degenerate eigenvalues at $\approx 11.0$ and $\approx 13.3$ were not resolved.

\begin{tabular}{rrrrrrr}
\hline$n$ & $m$ & $w_{\mathrm{ex}}$ & $w_{\mathrm{EBK}}$ & $m_{\mathrm{ex}}$ & $w_{\mathrm{hi}}$ & $m_{\mathrm{hi}}$ \\
\hline 0 & 0 & 2.404826 & 2.356194 & 1 & 2.356204 & 1.0005 \\
0 & 1 & 3.831706 & 3.794440 & 2 & 3.794444 & 1.9983 \\
0 & 2 & 5.135622 & 5.100386 & 2 & 5.100391 & 1.9996 \\
1 & 0 & 5.520078 & 5.497787 & 1 & 5.497785 & 0.9988 \\
0 & 3 & 6.380162 & 6.345186 & 2 & 6.345191 & 2.0000 \\
1 & 1 & 7.015587 & 6.997002 & 2 & 6.997006 & 2.0001 \\
0 & 4 & 7.588342 & 7.553060 & 2 & 7.553065 & 1.9992 \\
1 & 2 & 8.417244 & 8.400144 & 2 & 8.400149 & 1.9998 \\
2 & 0 & 8.653728 & 8.639380 & 1 & 8.639404 & 0.9987 \\
0 & 5 & 8.771484 & 8.735670 & 2 & 8.735677 & 2.0013 \\
1 & 3 & 9.761023 & 9.744628 & 2 & 9.744632 & 1.9999 \\
0 & 6 & 9.936110 & 9.899671 & 2 & 9.899675 & 1.9999 \\
2 & 1 & 10.173468 & 10.160928 & 2 & 10.160932 & 2.0000 \\
1 & 4 & 11.064709 & 11.048664 & 2 & 11.048968 & 4.0012 \\
0 & 7 & 11.086370 & 11.049268 & 2 & & \\
2 & 2 & 11.619841 & 11.608251 & 2 & 11.608256 & 2.0006 \\
3 & 0 & 11.791534 & 11.780972 & 1 & 11.780978 & 1.0001 \\
0 & 8 & 12.225092 & 12.187316 & 2 & 12.187319 & 1.9993 \\
1 & 5 & 12.338604 & 12.322723 & 2 & 12.322724 & 2.0000 \\
2 & 3 & 13.015201 & 13.004166 & 2 & 13.004168 & 1.9997 \\
3 & 1 & 13.323692 & 13.314197 & 2 & 13.315045 & 4.0287 \\
0 & 9 & 13.354300 & 13.315852 & 2 & & \\
1 & 6 & 13.589290 & 13.573465 & 2 & 13.573465 & 2.0000 \\
2 & 4 & 14.372537 & 14.361846 & 2 & 14.361849 & 1.9994 \\
0 & 10 & 14.475501 & 14.436391 & 2 & 14.436395 & 2.0006 \\
3 & 2 & 14.795952 & 14.787105 & 2 & 14.787076 & 1.9909 \\
1 & 7 & 14.821269 & 14.805435 & 2 & 14.805453 & 2.0066 \\
4 & 0 & 14.930918 & 14.922565 & 1 & 14.922569 & 1.0001 \\
\hline & & & & & &
\end{tabular}

in Ref. 24. We obtained similar results using only orbits up to length $s_{\max }=150$. This demonstrates the high efficiency of the harmonic inversion method in extracting eigenvalues from the periodic orbit sum. The efficiency can even be further increased with the help of cross-correlated periodic orbit sums as will be demonstrated in Section 5.4.

In Table 1 the exact multiplicities $m_{\mathrm{ex}}$ of eigenvalues and the multiplicities $m_{\mathrm{hi}}$ obtained by harmonic inversion also agree to very high precision. The deviations are one or two orders of magnitude larger than those in the frequencies, which reflects the fact that, with the harmonic inversion method using filter-diagonalization, the amplitudes usually converge more slowly than the frequencies.

In the frequency interval shown, there are two cases of nearly degenerate frequencies which have not been resolved by harmonic inversion of the periodic orbit signal with $s_{\max }=150$. The harmonic inversion yielded only one frequency, which is the average of the two nearly degenerate ones, with the amplitudes of the two added. These nearly degenerate states can be resolved when the signal 
length is increased to about $s_{\max }=500$ or with the help of cross-correlated periodic orbit sums (see Section 5.4).

\subsubsection{Semiclassical matrix elements}

Using the extended periodic orbit sums discussed in Section 2.3, we can now also calculate semiclassical diagonal matrix elements for the circle billiard.

Following the procedure described in Section 5.1, a semiclassical signal can be constructed from the extended response function, the analysis of which should again yield the eigenvalues $w_{k}$ as frequencies but with the amplitudes weighted with the diagonal matrix elements. As examples, we used the same operators as in Section 4.1.2 to calculate the diagonal matrix elements $\langle L\rangle,\langle r\rangle$, and the variance of the radius, $\left\langle r^{2}\right\rangle-\langle r\rangle^{2}$.

Figure 6 shows the results in the range $25 \leq w \leq 30$. For comparison, Fig. 6a presents the spectrum for the identity operator. The positions of the solid lines are the EBK eigenvalues, their heights are the semiclassical matrix elements obtained from EBK theory times the multiplicities. The crosses are the results of the harmonic inversion of a signal of length $s_{\max }=300$. The diagrams show excellent agreement between the results obtained by harmonic inversion and EBK torus quantization. This is even the case for the variance of $r$, which is a very small quantity.

For the states shown in Fig. 6 we have also compared the semiclassical to the exact quantum mechanical matrix elements. The agreement is also excellent. The deviations between the semiclassical and quantum matrix elements are typically of the order of $\sim 10^{-3}$, which can well be understood by the semiclassical approximation.

\subsection{Higher order $\hbar$ corrections}

The eigenvalues of the circle billiard obtained in the previous Section 5.2 are not the exact quantum mechanical eigenvalues but semiclassical approximations for the reason that the Berry-Tabor and the Gutzwiller formula are only the leading order terms of an expansion of the density of states in terms of $\hbar$ (see Section 2.4). We will now demonstrate how to obtain corrections to the semiclassical eigenvalues from the $\hbar$ expansion (39) of the periodic orbit sum

$$
g^{\mathrm{osc}}(w)=\sum_{n=0}^{\infty} g_{n}(w)=\sum_{n=0}^{\infty} \frac{1}{w^{n}} \sum_{\text {po }} \mathcal{A}_{\mathrm{po}}^{(n)} e^{i s_{\mathrm{po}} w}
$$

with $w=\hbar_{\text {eff }}^{-1}$ an effective inverse Planck constant (see Eq. (38)). The amplitudes $\mathcal{A}_{\text {po }}^{(0)}$ are those of the BerryTabor or Gutzwiller formula. For $n>0$, the amplitudes $\mathcal{A}_{\text {po }}^{(n)}$ (including also phase information) give the $n^{\text {th }}$ order corrections $g_{n}(w)$ to the response function $g^{\mathrm{osc}}(w)$. For
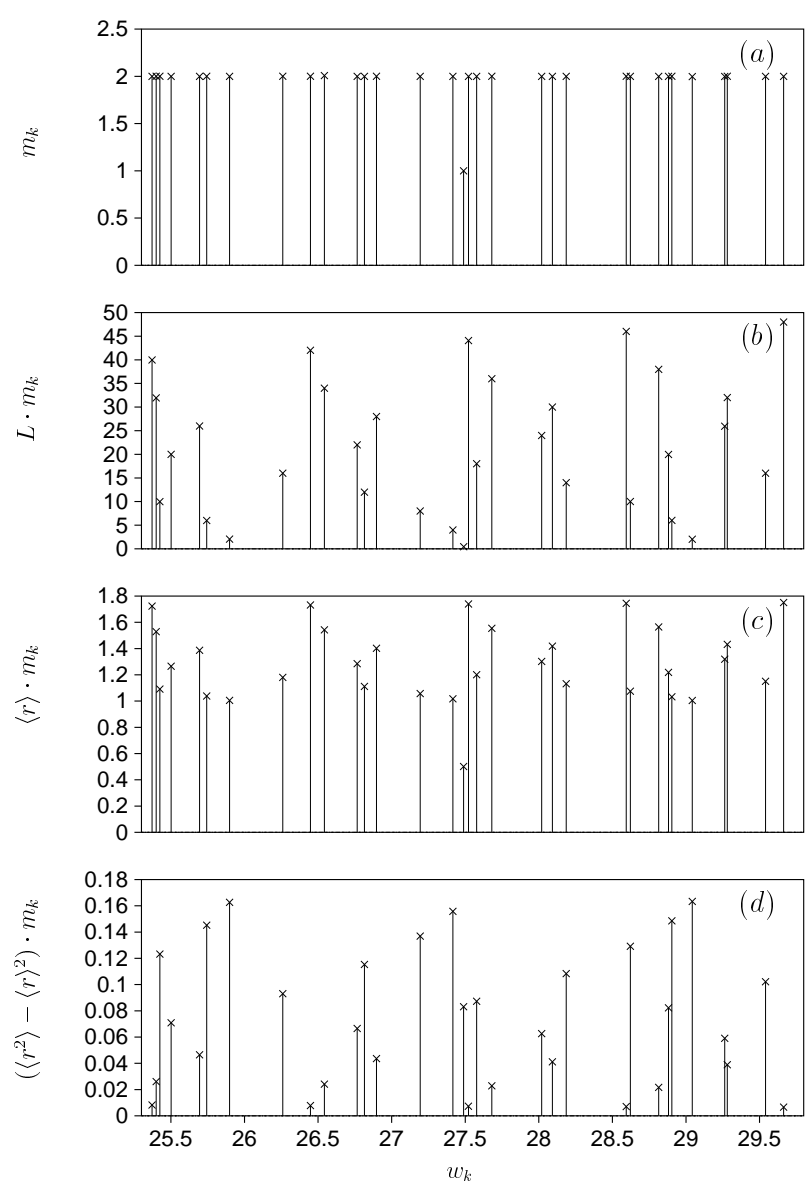

Fig. 6. Circle billiard: Results of the harmonic inversion of the semiclassical signal constructed from the periodic orbits sum including different operators. The quantities plotted are the diagonal matrix elements of the operators indicated times the multiplicities $m_{k}$, versus the eigenvalues $w_{k}$ of the scaling parameter. Crosses: results of the harmonic inversion procedure. Solid lines: semiclassical matrix elements obtained from EBK theory and EBK eigenvalues. $L$ : angular momentum in units of $\hbar, r$ : distance from the center in units of the radius $R$ of the billiard.

simplicity, we will assume in the following that the amplitudes $\mathcal{A}_{\text {po }}^{(n)}$ in $(39)$ do not depend on $w$. Again, in systems where the amplitudes possess a $w$ dependence of the form $\mathcal{A}_{\text {po }}^{(n)}=w^{\alpha} a_{\text {po }}^{(n)}$, the same line of arguments holds if we consider $g^{\prime}(w)=w^{-\alpha} g(w)$ instead of $g(w)$ (cf. Section 5.1).

For periodic orbit quantization the zeroth order contributions $\mathcal{A}_{\mathrm{po}}^{(0)}$ are usually considered only. As demonstrated in Section 5.1 (see Eqs. (77) and (78)), the Fourier transform of the principal periodic orbit sum

$$
C_{0}(s)=\sum_{\text {po }} \mathcal{A}_{\mathrm{po}}^{(0)} \delta\left(s-s_{\mathrm{po}}\right)
$$

is adjusted by application of the harmonic inversion technique to the functional form of the exact quantum expres- 
sion

$$
C_{\mathrm{qm}}(s)=-i \sum_{k} m_{k} e^{-i w_{k} s}
$$

with $\left\{w_{k}, m_{k}\right\}$ the eigenvalues and multiplicities.

For $n \geq 1$, the asymptotic expansion (39) of the semiclassical response function suffers from the singularities at $w=0$. It is therefore not appropriate to harmonically invert the Fourier transform of (39) as a whole, although the Fourier transform formally exists. This means that the method of periodic orbit quantization by harmonic inversion cannot straightforwardly be extended to the $\hbar$ expansion of the periodic orbit sum. Instead, we will calculate the correction terms to the semiclassical eigenvalues separately, order by order 115.

Let us assume that the $(n-1)^{\text {st }}$ order approximations $w_{k, n-1}$ to the semiclassical eigenvalues have already been obtained and the $w_{k, n}$ are to be calculated. The difference between the two subsequent approximations to the quantum mechanical response function reads

$$
\begin{aligned}
g_{n}(w) & =\sum_{k}\left(\frac{m_{k}}{w-w_{k, n}+i 0}-\frac{m_{k}}{w-w_{k, n-1}+i 0}\right) \\
& \approx \sum_{k} \frac{m_{k} \Delta w_{k, n}}{\left(w-\bar{w}_{k, n}+i 0\right)^{2}},
\end{aligned}
$$

with $\bar{w}_{k, n}=\frac{1}{2}\left(w_{k, n}+w_{k, n-1}\right)$ and $\Delta w_{k, n}=w_{k, n}-w_{k, n-1}$. Integration of (89) and multiplication by $w^{n}$ yields

$$
\mathcal{G}_{n}(w)=w^{n} \int g_{n}(w) d w=\sum_{k} \frac{-m_{k} w^{n} \Delta w_{k, n}}{w-\bar{w}_{k, n}+i 0}
$$

which has the functional form of a quantum mechanical response function but with residues proportional to the $n^{\text {th }}$ order corrections $\Delta w_{k, n}$ to the semiclassical eigenvalues. The semiclassical approximation to (90) is obtained from the term $g_{n}(w)$ in the periodic orbit sum (39) by integration and multiplication by $w^{n}$, i.e.,

$$
\begin{aligned}
\mathcal{G}_{n}(w) & =w^{n} \int g_{n}(w) d w \\
& =-i \sum_{\mathrm{po}} \frac{1}{s_{\mathrm{po}}} \mathcal{A}_{\mathrm{po}}^{(n)} e^{i w s_{\mathrm{po}}}+\mathcal{O}\left(\frac{1}{w}\right) .
\end{aligned}
$$

We can now Fourier transform both (90) and (91), and obtain $(n \geq 1)$

$$
\begin{aligned}
C_{n}(s) & \equiv \frac{1}{2 \pi} \int_{-\infty}^{+\infty} \mathcal{G}_{n}(w) e^{-i w s} d w \\
& =i \sum_{k} m_{k}\left(\bar{w}_{k}\right)^{n} \Delta w_{k, n} e^{-i \bar{w}_{k} s} \\
& \stackrel{\text { h.i. }}{=}-i \sum_{\text {po }} \frac{1}{s_{\mathrm{po}}} \mathcal{A}_{\mathrm{po}}^{(n)} \delta\left(s-s_{\mathrm{po}}\right) .
\end{aligned}
$$

Equations (92) and (93) imply that the $\hbar$ expansion of the semiclassical eigenvalues can be obtained, order by order, by harmonic inversion (h.i.) of the periodic orbit signal in

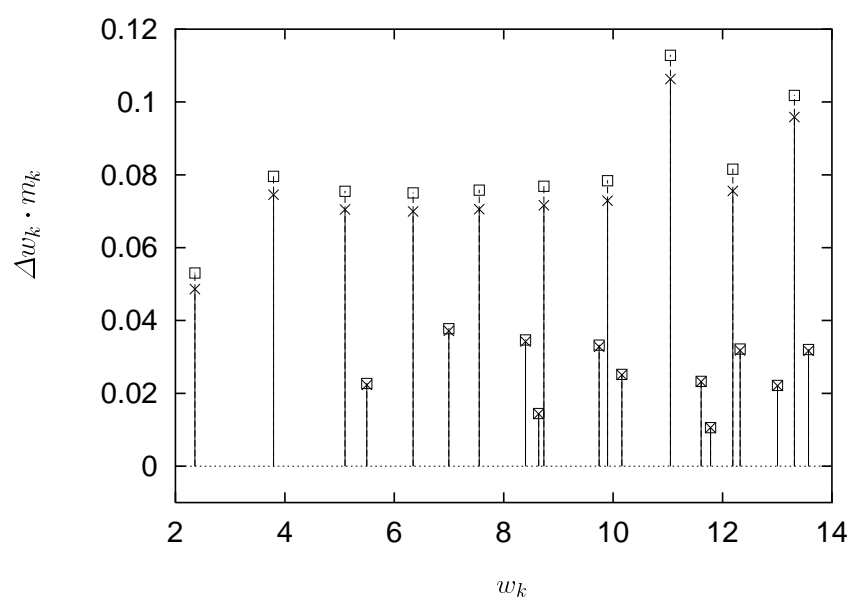

Fig. 7. Correction terms to the semiclassical eigenvalues of the circle billiard. Squares: corrections $\Delta w_{k, 1}=w_{k, 1}-w_{k, 0}$ between first and zeroth order approximations (times multiplicities) obtained by harmonic inversion. Crosses: differences $w_{\text {ex }}-w_{\text {EBK }}$ between exact quantum and EBK eigenvalues (times multiplicities).

(93) to the functional form of (92). [In practice, we will again convolute both expressions with a Gaussian function (cf. Section 5.1) in order to regularize the $\delta$ functions in (93).] The frequencies $\bar{w}_{k}$ of the periodic orbit signal (93) are the semiclassical eigenvalues, averaged over different orders of $\hbar$. Note that the accuracy of these values does not necessarily increase with increasing order $n$. We indicate this in (92) by omitting the index $n$ at the eigenvalues $\bar{w}_{k}$. Our numerical calculations for the first order $\hbar$ corrections show that, in practice, the frequencies $\bar{w}_{k}$ we obtain are approximately equal to the zeroth order $\hbar$ eigenvalues rather than the exact average between zeroth and first oder eigenvalues. The corrections $\Delta w_{k, n}$ to the eigenvalues are not obtained from the frequencies, but from the amplitudes, $m_{k}\left(\bar{w}_{k}\right)^{n} \Delta w_{k, n}$, of the periodic orbit signal.

We will now apply the above technique to the circle billiard to obtain the first order corrections to the semiclassical eigenvalues obtained in Section 5.2.2. In Section 2.2. we derived the zeroth order amplitudes of the circle billiard (cf. Eq. (26)):

$$
\frac{1}{\sqrt{w}} \mathcal{A}_{\mathrm{po}}^{(0)}=\sqrt{\frac{\pi}{2}} m_{\mathbf{M}} \frac{s_{\mathbf{M}}^{3 / 2}}{M_{r}^{2}} e^{-i\left(\frac{3}{2} M_{r} \pi+\frac{\pi}{4}\right)},
$$

with $s_{\mathbf{M}}$ and $m_{\mathbf{M}}$ the action and multiplicity of the orbit, respectively. The first order amplitudes are given by (cf. Sections 2.4 and 4.2):

$$
\frac{1}{\sqrt{w}} \mathcal{A}_{\mathrm{po}}^{(1)}=\sqrt{\pi M_{r}} \frac{2 \sin ^{2} \gamma-5}{6 \sin ^{3 / 2} \gamma} e^{-i\left(\frac{3}{2} M_{r} \pi-\frac{\pi}{4}\right)},
$$

with $\gamma \equiv \pi M_{\varphi} / M_{r}$.

Using these expressions, we have calculated the first order corrections $\Delta w_{k, 1}$ to the lowest eigenvalues of the circle billiard, by harmonic inversion of periodic orbit signals with $s_{\max }=200$. Part of the spectrum is presented in 


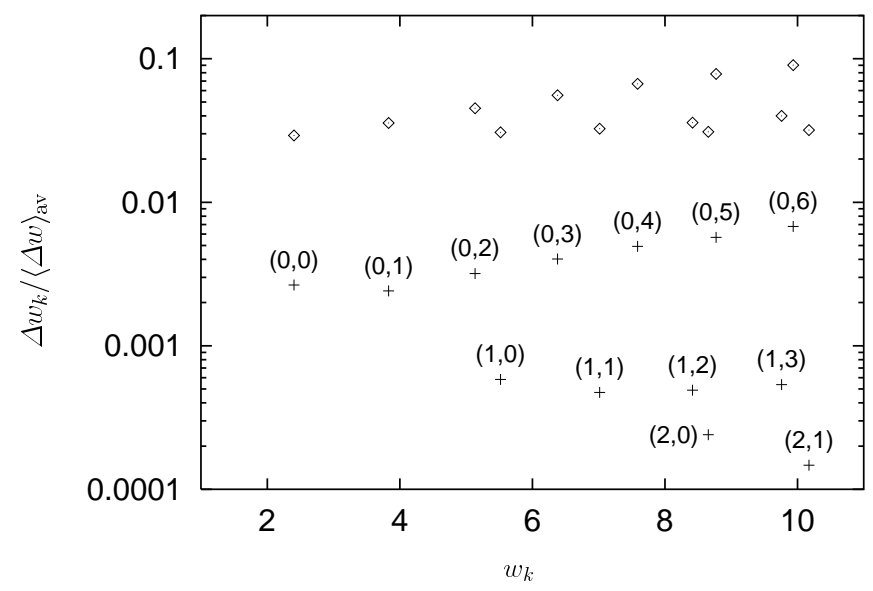

Fig. 8. Semiclassical errors $\left|w_{k, 0}-w_{\text {ex }}\right|$ (diamonds) and $\mid w_{k, 1}-$ $w_{\text {ex }} \mid$ (crosses) of zeroth and first order approximations to the eigenvalues obtained by harmonic inversion, in units of the average level spacing $\langle\Delta w\rangle_{\mathrm{av}} \approx 4 / w$. States are labeled by the quantum numbers $(n, m)$.

Figure 7. The peak heights (squares) are the corrections $\Delta w_{k, 1}=w_{k, 1}-w_{k, 0}$ times the multiplicities. For comparison, the differences between the exact and the EBK eigenvalues at the positions of the EBK eigenvalues are also plotted (see the crosses in Fig. 7). Both spectra are in excellent agreement. The small deviations of the peak heights arise from second or higher order corrections to the eigenvalues.

An appropriate measure for the accuracy of semiclassical eigenvalues is the deviation from the exact quantum eigenvalues in units of the average level spacings, $\langle\Delta w\rangle_{\mathrm{av}}=1 / \bar{\rho}(w)$. Figure 8 presents the semiclassical error in units of the average level spacings $\langle\Delta w\rangle_{\text {av }} \approx 4 / w$ for the zeroth order (diamonds) and first order (crosses) approximations to the eigenvalues. In zeroth order approximation the semiclassical error for the low lying states is about 3 to 10 percent of the mean level spacing. This error is reduced in the first order approximation by at least one order of magnitude for the least semiclassical states with radial quantum number $n=0$. The accuracy of states with $n \geq 1$ is improved by two or more orders of magnitude.

\subsection{Reduction of required signal length via harmonic}

\section{inversion of cross-correlated periodic orbit sums}

As described in the sections above, the harmonic inversion method is able to extract quantum mechanical eigenvalues from the semiclassical periodic orbit sum including periodic orbits up to a finite action $s_{\max }$. This means that in practice, although the periodic orbit sum does not converge, the eigenvalues can be obtained from a finite set of periodic orbits. The required signal length for harmonic inversion depends on the mean density of states, i.e., $s_{\max } \approx 4 \pi \bar{\rho}(w)$ (cf. (43)). Depending on the mean density of states, the action $s_{\max }$ up to which the periodic orbits have to be known may therefore be large. Due to the rapid proliferation of the number of periodic orbits with growing action, the efficiency and practicability of the procedure depends sensitively on the signal length required. This is especially the case when the periodic orbits have to be found numerically.

The quantization method can be improved with the help of cross-correlated periodic orbit sums. The extended response functions weighted with products of diagonal matrix elements discussed in Section 2.3, in combination with the method for harmonic inversion of cross-correlation functions presented in Section 3.2, can be used to significantly reduce the signal length required for the periodic orbit quantization [16,17. This technique is particularly helpful for chaotic systems, where the periodic orbits must be found numerically and where the number of periodic orbits grows exponentially with their action. However, for regular systems the number of orbits which have to be included can also be significantly reduced as will be demonstrated for the circle billiard.

The basic idea is to construct a set of signals where each individual signal contains the same frequencies (i.e., semiclassical eigenvalues) and the amplitudes are correlated by obeying the restriction (53). This can be achieved with the help of the generalized periodic orbit sum (34) introduced in Section 2.3:

A set of operators $A_{\alpha}, \alpha=1, \ldots, N$ is chosen. Following the procedure described in Section 5.1, the signals $C_{\alpha \alpha^{\prime}}(s)$ are obtained as Fourier transform of the generalized response functions $g_{\alpha \alpha^{\prime}}^{\mathrm{osc}}(w)$, i.e.,

$$
\begin{aligned}
g_{\alpha \alpha^{\prime}}^{\mathrm{osc}}(w) & =\sum_{\mathrm{po}} \mathcal{A}_{\mathrm{po}} \bar{A}_{\alpha, p} \bar{A}_{\alpha^{\prime}, p} e^{i s_{\mathrm{po}} w}, \\
C_{\alpha \alpha^{\prime}}(s) & =\sum_{\mathrm{po}} \mathcal{A}_{\mathrm{po}} \bar{A}_{\alpha, p} \bar{A}_{\alpha^{\prime}, p} \delta\left(s-s_{\mathrm{po}}\right),
\end{aligned}
$$

where for integrable systems the means $\bar{A}_{\alpha, p}$ are defined by (32). According to Sections 2.3 and 5.1, the corresponding quantum mechanical signal is given by

$$
C_{\mathrm{qm}, \alpha \alpha^{\prime}}(s)=-i \sum_{k} m_{k}\left\langle k\left|\hat{A}_{\alpha}\right| k\right\rangle\left\langle k\left|\hat{A}_{\alpha^{\prime}}\right| k\right\rangle e^{-i s w_{k}},
$$

where the amplitudes have the required form (53). As in Section 5.1 the semiclassical eigenvalues $w_{k}$ are obtained by adjusting the periodic orbit signal (97) (after convolution with a Gaussian function) to the functional form of the cross-correlated quantum signal (98) with the important difference that we now apply the extension of harmonic inversion to cross-correlation functions (see Section 3.2).

For the circle billiard, the mean density of states - with all multiplicities taken as one - is given by $\bar{\rho}(w)=w / 4$. According to (43), the signal length required for a single signal to resolve the frequencies in a given interval around $w$ is therefore approximately given by

$$
s_{\max } \approx 4 \pi \bar{\rho}(w)=\pi w=2 S_{H},
$$


Table 2. Nearly degenerate eigenvalues of the circle billiard, obtained by harmonic inversion of a $2 \times 2$ cross-correlated signal of length $s_{\max }=150$. The denotations are the same as in Table 11. The nearly degenerate eigenvalues are now resolved, which for a single signal would have required a signal length of $s_{\max } \approx 500$.

\begin{tabular}{rrrrrrr}
\hline$n$ & $m$ & $w_{\text {ex }}$ & $w_{\text {EBK }}$ & $m_{\text {ex }}$ & $w_{\mathrm{hi}}$ & $m_{\mathrm{hi}}$ \\
\hline 1 & 4 & 11.064709 & 11.048664 & 2 & 11.048664 & 2.0665 \\
0 & 7 & 11.086370 & 11.049268 & 2 & 11.049295 & 1.9315 \\
3 & 1 & 13.323692 & 13.314197 & 2 & 13.314205 & 1.9987 \\
0 & 9 & 13.354300 & 13.315852 & 2 & 13.315839 & 2.0016 \\
\hline
\end{tabular}

where $S_{H}=2 \pi \bar{\rho}$ is the Heisenberg period (which is action instead of time for scaling systems). By using an $N \times N$ set of signals, it should be possible to extract about the same number of semiclassical eigenvalues from a reduced signal length $s_{\max } \ll 2 S_{H}$, or, vice versa, if the signal length is held constant, the resolution and therefore the number of converged eigenvalues should significantly increase.

To demonstrate the power of the cross-correlation technique, we first analyze a $2 \times 2$ cross-correlated periodic orbit signal of the circle billiard with $\hat{A}_{1}=1$ the identity operator and $\hat{A}_{2}=r$. For comparison with the results in Section 5.2.2 we choose the same signal length $s_{\max }=150$. By contrast to the eigenvalues in Table 1 obtained from the one-dimensional signal the nearly degenerate states around $w \approx 11.05$ and $w \approx 13.3$ are now resolved as can be seen in Table 2. Note that a signal length $s_{\max } \approx 500$ is required to resolve these states without application of the cross-correlation technique.

As in all other calculations concerning cross-correlated signals, the results were improved by not making a sharp cut at the accumulation points but by damping the amplitudes of the orbits near these points. With the same cut-off criterion at the accumulation points, the total number of orbits in the signal with $s_{\max }=150$ was about 10 times smaller than in the signal with $s_{\max }=500$. This means, we could reduce the required number of orbits by one order of magnitude. For chaotic systems, where the number of orbits grows more rapidly (exponentially) with the maximum action, the improvement in the required number of orbits may even be better.

We now investigate the number of eigenvalues which do converge for fixed signal length but different sets and dimension of the cross-correlation matrix. Indeed, the highest eigenvalue $w_{\max }$ which can be resolved increases significantly when the cross-correlation technique is applied. However, the detailed results depend on the operators chosen. Furthermore, with increasing dimension of the matrix, the range in which the transition from resolved to unresolved eigenvalues takes place becomes broader, and the amplitudes in this region become less well converged. Rough estimates of $w_{\max }$ for various sets of operators and fixed signal length $s_{\max }=150$ are given in Table 3. For some of the signals, we used the extension of the trace for-
Table 3. Maximum frequencies $w_{\max }$ up to which the spectrum could be resolved using a $N \times N$ cross-correlated signal. The $b_{\alpha}$ are the operators or functions of operators used to build the signal (see text). $r$ : distance from center, $L$ : angular momentum in units of $\hbar w$.

\begin{tabular}{l|r|l}
\hline$N$ & $w_{\max }$ & $b_{\alpha}$ \\
\hline 1 & 45 & 1 \\
2 & 65 & $1,\langle r\rangle$ \\
3 & 90 & $1,\langle r\rangle, L^{2}$ \\
4 & 120 & $1,\langle r\rangle, L^{2}, e^{-(\langle r\rangle-0.7)^{2} / 3}$ \\
5 & 130 & $1,\langle r\rangle, L^{2}, 1 /\langle r\rangle^{2}, e^{-(L-1)^{2} / 10}$ \\
\hline
\end{tabular}

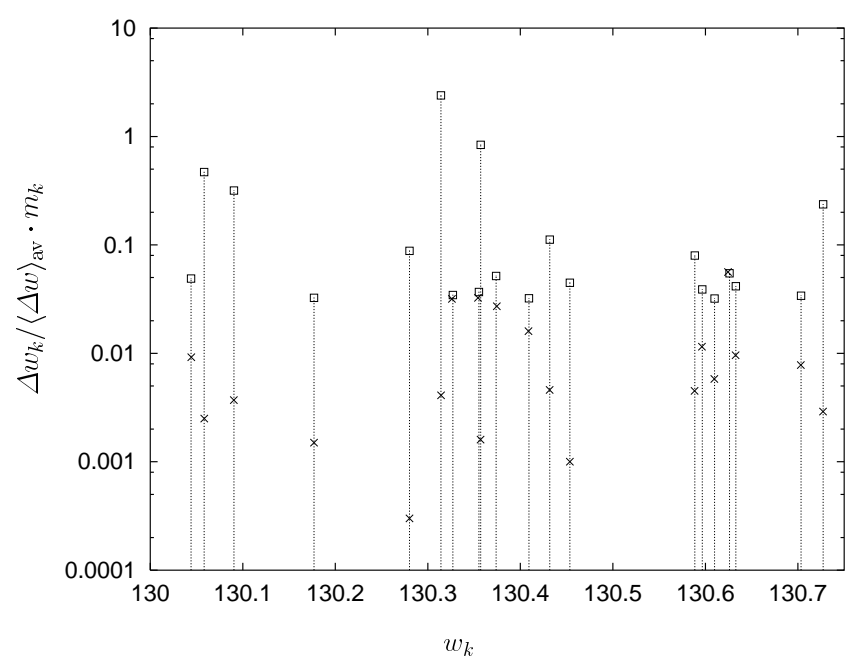

Fig. 9. Eigenvalues of the circle billiard in the dense part of the spectrum obtained from a $5 \times 5$ cross-correlated signal of length $s_{\max }=150=0.735 S_{H}$ with $S_{H}$ the Heisenberg period. With a single signal the required signal length would be $s_{\max } \approx 2 S_{H}$. Squares and sticks: EBK eigenvalues. Crosses: results of harmonic inversion. The peak heights give the semiclassical error $\left|w_{\mathrm{EBK}}-w_{\mathrm{ex}}\right|$ (squares) and the difference between harmonic inversion results and EBK eigenvalues $\left|w_{\mathrm{hi}}-w_{\mathrm{EBK}}\right|$ (crosses) in units of the mean level spacing $\langle\Delta w\rangle_{\text {av }} \approx 4 / w$. The error of the harmonic inversion procedure is about an order of magnitude smaller than the semiclassical error.

mula to functions of matrix elements, Eqs. (35) and (36). The improvement achieved by increasing the dimension of the matrix by one is most distinct for very small $N$; for $N \geq 5$, the improvement is only small. This suggests that, at given signal length and frequency range, the matrix dimension should not be chosen too large, i.e., there exists an optimal matrix dimension, which at constant signal length becomes larger with increasing eigenvalues $w$.

With a $5 \times 5$ signal of length $s_{\max }=150$, eigenvalues up to the region $w \approx 130$ can be resolved. The results are presented in Figure 9. There are two points which should be emphasized: The first point is that, even in this dense part of the spectrum, the error of the method is still by about one order of magnitude smaller than the semiclassical error, which is illustrated in Fig. 9 by the peak heights. The squares and crosses mark the semiclassical error 
$\left|w_{\mathrm{EBK}}-w_{\mathrm{ex}}\right|$ and the numerical error $\left|w_{\mathrm{hi}}-w_{\mathrm{EBK}}\right|$ of the harmonic inversion procedure in units of the mean level spacing $\langle\Delta w\rangle_{\mathrm{av}} \approx 4 / w$. The second point concerns the signal length compared to the Heisenberg action $S_{H}=2 \pi \bar{\rho}$. For $w=130$, one obtains $S_{H} \approx 204.2$. A one-dimensional signal would have required a signal length $s_{\max } \approx 2 S_{H}$. With the cross-correlation technique, we calculated the eigenvalues from a signal of length $s_{\max }=150 \approx 0.735 S_{H}$. This is about the same signal length as required by the semiclassical quantization method of Berry and Keating [10], which, however, only works for ergodic systems.

In summary, our results demonstrate that by analyzing cross-correlated signals instead of a single signal, the required signal length can indeed be significantly reduced. Clearly, the signal length cannot be made arbitrarily small, and the method is restricted to small dimensions of the cross-correlation matrix. However, the number of orbits which have to be included can be very much reduced. Another advantage of the method is that not only the frequencies and the multiplicities but also the diagonal matrix elements of the chosen operators are obtained by one single calculation.

\subsubsection{Including higher order $\hbar$ corrections}

In the cases discussed so far, we have constructed the cross-correlated signal by including different operators and making use of Eq. (34). By this procedure, we could obtain the semiclassical eigenvalues from a signal of reduced length or improve the resolution of the spectrum at constant signal length, while simultaneously obtaining the diagonal matrix elements of the operators. We can now even go one step further and include higher $\hbar$ corrections in the signal. Here we make use of the results of Section 5.3. The first order correction term, which in Section 5.3 was harmonically inverted as a single signal, is now included as part of a cross-correlated signal. This procedure combines all the techniques developed in the previous sections.

Formally, the frequencies in the zeroth and first order $\hbar$ parts of the cross-correlated signal are not exactly the same [see the denominators in Eqs. (75) and (90)], however, as already mentioned in Section 5.3, the numerically obtained values for the frequencies $\bar{w}_{k}$ in (92) are equal to the lowest order $\hbar$ eigenvalues rather than the exact average of the zeroth and first order eigenvalues. In practice, the cross-correlated signal is therefore in fact of the form (52). We can now, on the one hand, improve the resolution of the spectrum, and, on the other hand, obtain semiclassical matrix elements and the first order corrections to the eigenvalues with the same high resolution by one single harmonic inversion of a cross-correlated signal.

As an example, we built a $3 \times 3$ signal for the circle billiard from the first order correction term given by (95) and the operators $\hat{A}_{1}=\mathbf{1}$ (identity) and $\hat{A}_{2}=\hat{r}$. Again, we chose a signal length of $s_{\max }=150$. By harmonic inversion of the cross-correlated signal, we obtained simultaneously the semiclassical eigenvalues, their first order order corrections, and the semiclassical matrix elements of the op-
Table 4. Zeroth $\left(w_{k, 0}\right)$ and first $\left(w_{k, 1}\right)$ order semiclassical approximations to the eigenvalues of the circle billiard, obtained simultaneously by harmonic inversion of a $3 \times 3$ cross-correlated signal of length $s_{\max }=150$. The nearly degenerate eigenvalues at $w \approx 11.0$ and $w \approx 13.3$ are well resolved.

\begin{tabular}{rrrccc}
\hline$n$ & $m$ & $w_{\text {EBK }}$ & $w_{k, 0}$ & $w_{k, 1}$ & $w_{\text {ex }}$ \\
\hline 0 & 0 & 2.356194 & 2.356230 & 2.409288 & 2.404826 \\
0 & 1 & 3.794440 & 3.794440 & 3.834267 & 3.831706 \\
0 & 2 & 5.100386 & 5.100382 & 5.138118 & 5.135622 \\
1 & 0 & 5.497787 & 5.497816 & 5.520550 & 5.520078 \\
0 & 3 & 6.345186 & 6.345182 & 6.382709 & 6.380162 \\
1 & 1 & 6.997002 & 6.997006 & 7.015881 & 7.015587 \\
0 & 4 & 7.553060 & 7.553055 & 7.590990 & 7.588342 \\
1 & 2 & 8.400144 & 8.400145 & 8.417503 & 8.417244 \\
2 & 0 & 8.639380 & 8.639394 & 8.653878 & 8.653728 \\
0 & 5 & 8.735670 & 8.735672 & 8.774213 & 8.771484 \\
1 & 3 & 9.744628 & 9.744627 & 9.761274 & 9.761023 \\
0 & 6 & 9.899671 & 9.899660 & 9.938954 & 9.936110 \\
2 & 1 & 10.160928 & 10.160949 & 10.173568 & 10.173468 \\
1 & 4 & 11.048664 & 11.048635 & 11.063791 & 11.064709 \\
0 & 7 & 11.049268 & 11.049228 & 11.087943 & 11.086370 \\
2 & 2 & 11.608251 & 11.608254 & 11.619919 & 11.619841 \\
3 & 0 & 11.780972 & 11.780993 & 11.791599 & 11.791534 \\
0 & 8 & 12.187316 & 12.187302 & 12.228037 & 12.225092 \\
1 & 5 & 12.322723 & 12.322721 & 12.338847 & 12.338604 \\
2 & 3 & 13.004166 & 13.004167 & 13.015272 & 13.015201 \\
3 & 1 & 13.314197 & 13.314192 & 13.323418 & 13.323692 \\
0 & 9 & 13.315852 & 13.315782 & 13.356645 & 13.354300 \\
1 & 6 & 13.573465 & 13.573464 & 13.589544 & 13.589290 \\
2 & 4 & 14.361846 & 14.361846 & 14.372606 & 14.372537 \\
0 & 10 & 14.436391 & 14.436375 & 14.478531 & 14.475501 \\
3 & 2 & 14.787105 & 14.787091 & 14.795970 & 14.795952 \\
1 & 7 & 14.805435 & 14.805457 & 14.821595 & 14.821269 \\
4 & 0 & 14.922565 & 14.922572 & 14.930938 & 14.930918 \\
0 & 11 & 15.550089 & 15.550084 & 15.593060 & 15.589848 \\
2 & 5 & 15.689703 & 15.689701 & 15.700239 & 15.700174 \\
1 & 8 & 16.021889 & 16.021888 & 16.038034 & 16.037774 \\
3 & 3 & 16.215041 & 16.215047 & 16.223499 & 16.223466 \\
4 & 1 & 16.462981 & 16.462982 & 16.470648 & 16.470630 \\
0 & 12 & 16.657857 & 16.657846 & 16.701442 & 16.698250 \\
2 & 6 & 16.993489 & 16.993486 & 17.003884 & 17.003820 \\
1 & 9 & 17.225257 & 17.225252 & 17.241482 & 17.241220 \\
3 & 4 & 17.607830 & 17.607831 & 17.615994 & 17.615966 \\
0 & 13 & 17.760424 & 17.760386 & 17.804708 & 17.801435 \\
4 & 2 & 17.952638 & 17.952662 & 17.959859 & 17.959819 \\
5 & 0 & 18.064158 & 18.064201 & 18.071125 & 18.071064 \\
\hline & & & & & \\
\hline
\end{tabular}

erator $\hat{r}$. The results for the zeroth order approximations $w_{k, 0}$ to the eigenvalues and the first order approximations $w_{k, 1}=w_{k, 0}+\Delta w_{k, 1}$ are presented in Table 1 . For comparison the exact and the EBK eigenvalues are also given. As for the results presented in Table 2, we were able to resolve the nearly degenerate states in the zeroth order approximation, which for a single signal would have required a signal length of $s_{\max } \approx 500$. Moreover, in contrast to the results of Section 5.3, we could now also resolve the first order approximations to the nearly degenerate states. 


\section{Conclusion}

The harmonic inversion method has been introduced as a powerful tool for the calculation of quantum eigenvalues from periodic orbit sums as well as for the high resolution analysis of quantum spectra in terms of classical periodic orbits. We have demonstrated that this method, which has already successfully been applied to classically chaotic systems, yields excellent results for regular systems as well. Harmonic inversion has thus been shown to be a universal method, which, in contrast to other high resolution methods, does not depend on special properties of the system such as ergodicity or the existence of a symbolic code.

With the harmonic inversion method, we are able to calculate the contributions of the classical periodic orbits to the trace formula from the quantum eigenvalues with high precision and high resolution. By analyzing the difference spectrum between exact and semiclassical eigenvalues, we could determine higher order $\hbar$ corrections to the periodic orbit sum of the circle billiard.

Up to now, no theory for the $\hbar$ corrections to the BerryTabor formula for regular systems has been developed. We have numerically found an expression for the first order $\hbar$ corrections to the Berry-Tabor formula by harmonic inversion of the difference spectrum. The same expression can be derived analytically by using Vattay's and Rosenqvist's method for chaotic systems and introducing some reasonable ad-hoc assumption for the circle billiard. As this is clearly not a strict derivation, it is an interesting task for the future to develop a general theory for the higher order $\hbar$ corrections to the trace formula for regular systems.

In addition to calculating semiclassical eigenvalues from the usual periodic orbit sum, we have demonstrated how further information can be extracted from the parameters of the classical orbits by applying the harmonic inversion technique to different extensions of the trace formula. Using a generalized trace formula including an arbitrary operator, we have shown that the method also allows the calculation of semiclassical diagonal matrix elements from the parameters of the periodic orbits. Furthermore we have demonstrated how higher order $\hbar$ corrections to the semiclassical eigenvalues can be obtained by harmonic inversion of correction terms to the periodic orbit sums. For the case of the circle billiard, we found that, including the first order correction, the accuracy of the semiclassical eigenvalues compared to the exact quantum eigenvalues could be improved by one or more orders of magnitude.

Although by harmonic inversion the quantum eigenvalues can be calculated from a semiclassical signal of finite length, i.e., from a finite set of periodic orbits, the number of orbits which have to be included may still be large. We have demonstrated that by a generalization of the harmonic inversion method to cross-correlation functions the required signal length may be significantly reduced, even below the Heisenberg time. Because of the rapid proliferation of periodic orbits with growing period, this means that the number of orbits which have to be included may be reduced by about one to several orders of magnitude.

\section{A Calculation of the first order $\hbar$ correction terms to the semiclassical trace formula}

The calculation of higher order $\hbar$ corrections to the semiclassical eigenvalues introduced in Section 2.4 requires the knowledge of the $n$th order amplitudes $\mathcal{A}_{\mathrm{po}}^{(n)}$ in the periodic orbit sum (39). In this Appendix, we briefly outline the derivation of the first order amplitudes $\mathcal{A}_{\mathrm{po}}^{(1)}$ and the application to the circle billiard given in Eq. (40).

Two different methods for the calculation of higher order $\hbar$ correction terms in chaotic systems have been derived by Gaspard and Alonso 31,32 and Vattay and Rosenqvist [33, 34, 35]. The latter method has been specialized to two-dimensional chaotic billiards in 35. Here, we follow the approach of Vattay and Rosenquist. However, it is important to note that both methods cannot straightforwardly be applied to integrable systems and additional assumptios will be necessary to derive Eq. (40) for the circle billiard. A general theory for the calculation of higher order $\hbar$ corrections to the Berry-Tabor formula (4) for integrable systems is, to the best of our knowledge, still lacking.

Vattay and Rosenqvist give a quantum generalization of Gutzwiller's trace formula based on the path integral representation of the quantum propagator. The basic idea of their method is to express the global eigenvalue spectrum in terms of local eigenvalues computed in the neighbourhood of periodic orbits. The energy domain Green function $G\left(q, q^{\prime}, E\right)$ is connected to the spectral determinant $\Delta(E)=\Pi_{n}\left(E-E_{n}\right)$, with $E_{n}$ the energy eigenvalues or resonances, by

$$
\operatorname{Tr} G(E)=\int d q G(q, q, E)=\frac{d}{d E} \ln \Delta(E) .
$$

The trace of the Green function can be expressed in terms of contributions from periodic orbits

$$
\operatorname{Tr} G(E)=\sum_{\text {p.o. }} \operatorname{Tr} G_{p}(E),
$$

with the local traces connected to the local spectral determinants by

$$
\operatorname{Tr} G_{p}(E)=\frac{d}{d E} \ln \Delta_{p}(E)
$$

The trace of the Green function can therefore be calculated by solving the local Schrödinger equation around each periodic orbit, which yields the local eigenspectra.

To obtain the local eigenspectra, the ansatz

$$
\psi=\Phi e^{i S / \hbar}
$$

is inserted into the Schrödinger equation, yielding the following differential equations for $\Phi$ and $S$.

$$
\begin{aligned}
& \partial_{t} S+\frac{1}{2}(\nabla S)^{2}+U=0 \\
& \partial_{t} \Phi+\nabla \Phi \nabla S+\frac{1}{2} \Phi \Delta S-\frac{i \hbar}{2} \Delta \Phi=0
\end{aligned}
$$


where $U$ is the potential entering the Schrödinger equation.

The spectral determinant can be calculated from the local eigenvalues of the amplitudes $\Phi$. For arbitrary energy E, the amplitudes $\Phi_{p}^{l}$ of the local eigenfunctions fulfill the equation

$$
\Phi_{p}^{l}\left(t+T_{p}\right)=R_{p}^{l}(E) \Phi_{p}^{l}(t)
$$

where $T_{p}$ is the period of the classical orbit. Using Eq. (102), the trace formula can be expressed in terms of the eigenvalues $R_{p}^{l}(E)$ :

$$
\begin{aligned}
\operatorname{Tr} G(E)=\frac{1}{i \hbar} & \sum_{p} \sum_{l}\left(T_{p}(E)-i \hbar \frac{d \ln R_{p}^{l}(E)}{d E}\right) \\
& \times \sum_{r=1}^{\infty}\left(R_{p}^{l}(E)\right)^{r} e^{\frac{i}{\hbar} r S_{p}(E)} .
\end{aligned}
$$

This is the quantum generalization of Gutzwiller's trace formula and holds exactly.

The amplitudes and their eigenvalues are now expanded in powers of $\hbar$ :

$$
\begin{aligned}
\Phi^{l} & =\sum_{m=0}^{\infty}\left(\frac{i \hbar}{2}\right)^{m} \Phi^{l(m)} \\
R^{l}(E) & =\exp \left\{\sum_{m=0}^{\infty}\left(\frac{i \hbar}{2}\right)^{m} C_{l}^{(m)}\right\} \\
& \approx \exp \left(C_{l}^{(0)}\right)\left(1+\frac{i \hbar}{2} C_{l}^{(1)}+\ldots\right)
\end{aligned}
$$

The terms $C_{l}^{(0)}$ yield the Gutzwiller trace formula as zeroth order approximation, while the terms with $m>0$ give $\hbar$ corrections.

To solve Eqs. (104) and (105) in different order of $\hbar$, the Schrödinger equation and the functions $\Phi^{l(m)}$ and $S$ are Taylor expanded around the periodic orbit,

$$
\begin{aligned}
S(\mathbf{q}, t) & =\sum \frac{1}{\mathbf{n} !} s_{\mathbf{n}}(t)\left(\mathbf{q}-\mathbf{q}_{p}(t)\right)^{\mathbf{n}} \\
\Phi^{l(m)}(\mathbf{q}, t) & =\sum \frac{1}{\mathbf{n} !} \phi_{\mathbf{n}}^{l(m)}(t)\left(\mathbf{q}-\mathbf{q}_{p}(t)\right)^{\mathbf{n}},
\end{aligned}
$$

resulting in a set of differential equations for the different orders of the Taylor expansions and different orders in $\hbar$. In one dimension, these equations read explicitly:

$$
\dot{s}_{n}-s_{n+1} \dot{q}+\frac{1}{2} \sum_{k=0}^{n} \frac{n !}{(n-k) ! k !} s_{n-k+1} s_{k+1}+u_{n}=0,
$$

where $u_{n}$ are the coefficients of the Taylor expanded potential, and

$$
\begin{aligned}
\dot{\phi}_{n}^{(m+1)}- & \phi_{n+1}^{(m+1)} \dot{q} \\
+ & \sum_{k=0}^{n} \frac{n !}{(n-k) ! k !} \times \\
& \left(\phi_{n-k+1}^{(m+1)} s_{k+1}+\frac{1}{2} \phi_{n-k}^{(m+1)} s_{k+2}\right) \\
& -\phi_{n+2}^{(m)}=0
\end{aligned}
$$

This set of differential equations can be solved iteratively. The $l$-th eigenfunction is characterized by the condition $\phi_{n}^{(m)} \equiv 0$ for $n<l$. The different orders of $\hbar$ are connected by the last term in (114). Starting from zeroth order $\hbar$ and the lowest nonvanishing order of the Taylor expansion, the functions can be determined order by order. For higher dimensional systems, the coefficient matrices obey similar equations, and the structure of the set of equations remains the same.

To obtain the first order $\hbar$ correction to the Gutzwiller trace formula, one has to calculate the quantities $C_{l}^{(1)}$. To obtain these quantities one has to solve the set of equations (114) up to the lowest nonvanishing first order $\hbar$ coefficient function $\phi_{l}^{l(1)}$, respectively. As $\phi_{n}^{l(m)} \equiv 0$ for $n<l$, this involves solving the equations for $s_{2}, s_{3}$ and $s_{4}$, and for the zeroth order $\hbar$ coefficient functions $\phi_{l}^{l(0)}, \phi_{l+1}^{l(0)}$ and $\phi_{l+2}^{l(0)}$. If the initial conditions are set to be $\Phi_{l}^{l(0)}(0)=1$ and $\Phi_{l}^{l(m)}(0)=0$ for $m>0$, the correction term $C_{l}^{(1)}$ is then given by the relation

$$
C_{l}^{(1)}=\frac{\phi_{l}^{l(1)}\left(T_{p}\right)}{\exp \left(C_{l}^{(0)}\right)}
$$

which follows from the $\hbar$ expansion of the eigenequation (106).

An explicit recipe for the calculation of the first $\hbar$ correction for two-dimensional chaotic billiards is given in [35]. For billiards, the potential $U$ in the Schrödinger equation equals zero between two bounces at the hard wall. The functions $S$ and $\Phi$ now have to be Taylor expanded in two dimensions:

$$
\begin{aligned}
S(x, y, t) & =S_{0}+S_{x} \Delta x+S_{y} \Delta y \\
& +\frac{1}{2}\left(S_{x^{2}}(\Delta x)^{2}+2 S_{x y} \Delta x \Delta y+S_{y^{2}}(\Delta y)^{2}\right) \\
& +\ldots
\end{aligned}
$$

and similarly for $\Phi$. If the coordinate system is chosen in such a way that $x$ is along the periodic orbit and $y$ is perpendicular to the orbit, derivatives with respect to $x$ can be expressed in terms of the derivatives with respect to $y$ using the stationarity conditions

$$
S_{x^{n+1} y^{m}}=\frac{\dot{S}_{x^{n} y^{m}}}{S_{x}}, \quad \phi_{x^{n+1} y^{m}}=\frac{\dot{\phi}_{x^{n} y^{m}}}{S_{x}} .
$$

The quantity $S_{x}$ is equal to the classical momentum of the particle.

For the free motion between the collisions with the wall, the set of differential equations correspoding to 113 and (114) then reduces to a set of equations involving only derivatives with respect to $y$. These equations can be solved analytically, with the general solution still containing free parameters. Setting $S_{x}=1$, the first coefficient functions of the Taylor expanded phase are given by:

$$
S_{y y}(t)=\frac{1}{t+t_{0}}
$$




$$
\begin{aligned}
S_{y y y}(t) & =\frac{A}{\left(t+t_{0}\right)^{3}} \\
S_{y y y y}(t) & =-\frac{3}{\left(t+t_{0}\right)^{3}}+\frac{B}{\left(t+t_{0}\right)^{4}}+\frac{3 A^{2}}{\left(t+t_{0}\right)^{5}}
\end{aligned}
$$

where $t_{0}, A$ and $B$ are free parameters. For given $l$, the first nonvanishing coefficients of the amplitude read

$$
\begin{aligned}
\phi_{y^{l}}^{(0)}(t)= & E\left(\frac{t_{0}}{t+t_{0}}\right)^{l+1 / 2} \\
\phi_{y^{l+1}}^{(0)}(t)= & \frac{E}{\left(t+t_{0}\right)^{l+3 / 2}}\left[C+(l+1)^{2} \frac{A}{2} \frac{t_{0}^{l+1 / 2}}{\left(t+t_{0}\right)}\right] \\
\phi_{y^{l+2}}^{(0)}(t)= & \frac{E}{\left(t+t_{0}\right)^{l+5 / 2}}\left\{D+\frac{1}{t+t_{0}} \times\right. \\
& {\left[(l+2)^{2} \frac{A C}{2}+(l+2)(l+1)\left(\frac{l}{3}+\frac{1}{2}\right) \frac{B}{2} t_{0}^{l+1 / 2}\right] } \\
+ & \frac{A^{2} t_{0}^{l+1 / 2}}{2\left(t+t_{0}\right)^{2}} \times \\
& {\left.\left[\frac{1}{4}(l+2)^{2}(l+1)^{2}+\frac{3}{2}(l+2)(l+1)\left(\frac{l}{3}+\frac{1}{2}\right)\right]\right\} }
\end{aligned}
$$

Again, $D$ and $E$ are free parameters.

At the collisions with the hard wall, the phase and amplitude have to obey the bouncing conditions

$$
\begin{aligned}
& S\left(x, y, t_{-0}\right)=S\left(x, y, t_{+0}\right)+i \pi \\
& \Phi\left(x, y, t_{-0}\right)=\Phi\left(x, y, t_{+0}\right)
\end{aligned}
$$

from which the bouncing conditions for the coefficients of the Taylor expanded functions $S$ and $\Phi$ can be derived. While the general solutions between the bounces are valid for all billiards, the bouncing conditions in their Taylor expanded form depend explicitely on the shape of the hard wall.

An additional condition which the solutions $S$ and $\Phi$ have to obey is periodicity along the orbit. With every traversal the phase gains the same constant contributions at the collisions with the wall. The derivatives of the phase are periodic. The amplitude collects the same factor with each traversal, which means that all Taylor coefficients of the amplitude are periodic apart from a constant factor. These conditions together with the bouncing conditions determine the values of the free constants in the general solutions between the collisions.

The solutions can in general be found numerically, by choosing suitable initial conditions and following the evolution of the phase and amplitude functions along the orbit. After several iterations around the orbit, the parameters should converge against their periodic solution. The correction terms $C_{l}^{(1)}$ are then given by the integral

$$
C_{l}^{(1)}=\int \frac{\phi_{y^{l+2}}^{l(0)}+\phi_{y^{l} x^{2}}^{l(0)}}{\phi_{y^{l}}^{l(0)}} d t,
$$

which can be computed explicitly from the solutions found above.

As already explained, this method is designed for chaotic systems, as its derivation is based on the assumption that the periodic orbits are isolated. Nevertheless, we obtain reasonable results when applying the method to the circle billiard, taking one periodic orbit from each rational torus and introducing some additional assumptions.

Because of the symmetry of the orbits, we can assume that every side of the orbit contributes in the same manner to the $\hbar$ correction term for the whole orbit. This means, if we reset $t=0$ at the start of each side, the free parameters in the general solutions (118) to (123) must be the same for each side, apart from the parameter $E$, which collects the same factor during every collison with the wall. With these assumptions, the differential equations can be solved analytically.

However, it turns out that the bouncing conditions resulting from (124) and (125) are not sufficient to determine all free parameters, as some of the conditions are automatically fulfilled. We need additional conditions for the parameters. These can be obtained from the rotational symmetry of the system: Because of this symmetry, we can assume that the amplitude of the wave function does not depend on the polar angle $\varphi$. The same holds for all derivatives of the amplitude with respect to the radius $r$. For the zeroth order $\hbar$ amplitudes, expressed in polar coordinates $(r, \varphi)$, this gives us the additional conditions we need:

$$
\frac{\partial}{\partial \varphi} \frac{\partial^{n} \Phi^{l(0)}}{\partial r^{n}}=0
$$

If we further assume that the phase separates in polar coordinates

$$
S(r, \varphi)=S_{r}(r)+S_{\varphi}(\varphi)
$$

which implies that all mixed derivatives vanish, it turns out that we do not need the bouncing conditions at all. All parameters can be determined from the symmetry of the system, and the bouncing conditions are automatically fulfilled. We considered only the case $l=0$, for which we used Eq. (128) together with the conditions

$$
\frac{\partial}{\partial \varphi} \Phi^{0(0)}=0, \quad \frac{\partial}{\partial \varphi} \frac{\partial \Phi^{0(0)}}{\partial r}=0 .
$$

Our final results for the constant parameters in Eqs. (118) to $(123)$ are

$$
\begin{aligned}
t_{0} & =-\sin \gamma \\
A & =-\cos \gamma \\
B & =0 \\
C & =0 \\
D & =-\frac{i}{2} \sin ^{1 / 2} \gamma
\end{aligned}
$$

with $\gamma$ as defined in Section 2.4 (see Fig. 1). The radius of the billiard was taken to be $R=1$. Inserting these solutions in (126) finally leads to

$$
C_{0}^{(1)}=M_{r}\left(\frac{1}{3 \sin \gamma}-\frac{5}{6 \sin ^{3} \gamma}\right)
$$


where $M_{r}$ is the number of sides of the orbit.

The first order amplitudes $\mathcal{A}^{(1)}$ are obtained by inserting the $\hbar$ expansion (110) in the trace formula (107) and comparing the result with the $\hbar$ expansion (39). In the units we have used here (radius $R=1$ and momentum $\hbar k=1$ ), the scaling parameter $w$ is equal to $\hbar$. If we use only the $l=0$ contributions and assume that the terms $\exp \left(C_{0}^{(0)}\right)$ are equal to the amplitudes given by the Berry-Tabor formula, we finally end up with the expression (40). Although we cannot strictly justify the last step, our analysis of the quantum spectrum in Section 4.2 provides strong numerical evidence that Eq. (40) is correct. It will be an interesting task for the future to develop a general theory for the $\hbar$ correction terms of integrable systems and thus to provide a more rigorous mathematical proof of Eq. (40).

\section{Acknowledgements}

This work was supported by the Deutsche Forschungsgemeinschaft.

\section{References}

1. A. Einstein, Verh. Dtsch. Phys. Ges. 19, (1917) 82.

2. L. Brillouin, J. Phys. Radium 7, (1926) 353.

3. J. B. Keller, Ann. Phys. (N.Y.) 4, (1958) 180.

4. I. C. Percival, Adv. Chem. Phys. 36, (1977) 1.

5. M. V. Berry and M. Tabor, Proc. R. Soc. Lond. A 349, 101 (1976); J. Phys. A 10, (1977) 371.

6. M. C. Gutzwiller, J. Math. Phys. 8, (1967) 1979; 12, (1971) 343.

7. M. C. Gutzwiller, Chaos in Classical and Quantum Mechanics (Springer, New York, 1990).

8. P. Cvitanović and B. Eckhardt, Phys. Rev. Lett. 63, (1989) 823.

9. R. Aurich, C. Matthies, M. Sieber, and F. Steiner, Phys. Rev. Lett. 68, (1992) 1629.

10. M. V. Berry and J. P. Keating, J. Phys. A 23, 4839 (1990); Proc. R. Soc. Lond. A 437, (1992) 151.

11. J. Main, V. A. Mandelshtam, and H. S. Taylor, Phys. Rev. Lett. 79, (1997) 825.

12. J. Main, V. A. Mandelshtam, G. Wunner, and H. S. Taylor, Nonlinearity 11, (1998) 1015.

13. J. Main, Phys. Rep. 316, (1999) 233.

14. J. Main and G. Wunner, Phys. Rev. Lett. 82, (1999) 3038.

15. J. Main, K. Weibert, and G. Wunner, Phys. Rev. E 58, (1998) 4436.

16. J. Main and G. Wunner, Phys. Rev. E 60, (1999) 1630.

17. J. Main, K. Weibert, V. A. Mandelshtam, and G. Wunner, Phys. Rev. E 60, (1999) 1639.

18. S. Hortika and M. Srednicki, Phys. Rev. E 61, (2000) in press.

19. M. R. Wall and D. Neuhauser, J. Chem. Phys. 102, (1995) 8011.

20. V. A. Mandelshtam and H. S. Taylor, Phys. Rev. Lett. 78, (1997) 3274; J. Chem. Phys. 107, (1997) 6756.

21. E. Narevicius, D. Neuhauser, H. J. Korsch, and N. Moiseyev, Chem. Phys. Lett. 276, (1997) 250.
22. V. A. Mandelshtam, J. Chem. Phys. 108, (1998) 9999.

23. J. Main, V. A. Mandelshtam, and H. S. Taylor, Phys. Rev. Lett. 78, (1997) 4351.

24. S. M. Reimann, M. Brack, A. G. Magner, J. Blaschke, and M. V. N. Murthy, Phys. Rev. A 53, (1996) 39.

25. D. Ullmo, M. Grinberg, and S. Tomsovic, Phys. Rev. E 54, (1996) 136.

26. B. Mehlig, Phys. Rev. E 59, (1999) 390.

27. R. Balian and C. Bloch, Ann. Phys. 69, (1972) 76.

28. M. Wilkinson, J. Phys. A 20, (1987) 2415.

29. M. Wilkinson, J. Phys. A 21, (1988) 1173.

30. B. Eckhardt, S. Fishman, K. Müller, and D. Wintgen, Phys. Rev. A 45, (1992) 3531.

31. D. Alonso and P. Gaspard, Chaos 3, (1993) 601.

32. P. Gaspard and D. Alonso, Phys. Rev. A 47, (1993) R3468.

33. G. Vattay, chao-dyn/9406005, unpublished (1994).

34. G. Vattay and P. Rosenqvist, Phys. Rev. Lett. 76, (1996) 335.

35. P. Rosenqvist, Ph.D. thesis, unpublished (1994). 